Article

\title{
A Camera-Based Target Detection and Positioning UAV System for Search and Rescue (SAR) Purposes
}

\author{
Jingxuan Sun, Boyang Li, Yifan Jiang and Chih-yung Wen * \\ Department of Mechanical Engineering, The Hong Kong Polytechnic University, Hong Kong, China; \\ jingxuan.j.sun@connecy.polyu.hk (J.S.); boyang.li@connect.polyu.hk (B.L.); \\ jiang.uhrmacher@connect.polyu.hk (Y.J.) \\ * Correspondence: cywen@polyu.edu.hk; Tel.: +852-2766-6644 \\ Academic Editors: Felipe Gonzalez Toro and Antonios Tsourdos \\ Received: 30 August 2016; Accepted: 19 October 2016; Published: 25 October 2016
}

\begin{abstract}
Wilderness search and rescue entails performing a wide-range of work in complex environments and large regions. Given the concerns inherent in large regions due to limited rescue distribution, unmanned aerial vehicle (UAV)-based frameworks are a promising platform for providing aerial imaging. In recent years, technological advances in areas such as micro-technology, sensors and navigation have influenced the various applications of UAVs. In this study, an all-in-one camera-based target detection and positioning system is developed and integrated into a fully autonomous fixed-wing UAV. The system presented in this paper is capable of on-board, real-time target identification, post-target identification and location and aerial image collection for further mapping applications. Its performance is examined using several simulated search and rescue missions, and the test results demonstrate its reliability and efficiency.
\end{abstract}

Keywords: unmanned aerial vehicle (UAV); wilderness search and rescue; target detection

\section{Introduction}

Wilderness search and rescue (SAR) is challenging, as it involves searching large areas with complex terrain for a limited time. Common wilderness search and rescue missions include searching and rescuing injured humans and finding broken and lost cars in deserts, forests or mountains. Incidents of commercial aircraft disappearing from radar, such as the case in Indonesia in 2014 [1-3], also entail a huge search radius and search timeliness is critical to "the probability of finding and successfully aiding the victim" [4-7]. This research focuses on applications common in eastern Asian locations such as Hong Kong, Taiwan, the southeastern provinces of mainland China, Japan and the Philippines, where typhoons and earthquakes happen a few times annually, causing landslides and river flooding that result in significant damage to houses, roads and human lives. Immediate assessment of the degree of damage and searching for survivors are critical requirements for constructing a rescue and revival plan. UAV-based remote image sensing can play an important role in large-scale SAR missions $[4-6,8,9]$.

With the development of micro-electro-mechanical system (MEMS) sensors, the use of small UAVs (with a wing-span of under $10 \mathrm{~m}$ ) is a promising platform for conducting search, rescue and environmental surveillance missions. UAVs can be equipped with various remote sensing systems, such as powerful tools for observing disaster mitigation, including rapid all-weather flood and earthquake damage assessment. Today, low price drones allow people to quickly develop small UAVs, which have the following specific advantages:

- Can loiter for lengthy periods at preferred altitudes;

- Produce remote sensor data with better resolution than satellites, particularly in terms of image quality; 
- Low cost, rapid response;

- Capable of flying below normal air traffic height;

- Can get closer to areas of interest.

Applying UAV technology and remote sensing to search, rescue and environmental surveillance is not a new idea. Habib et al. stated the advantages of applying UAV technologies to surveillance, security and mission planning, compared with the normal use of satellites, and various technologies and applications have been integrated and tested on UAV-assisted operations [9-13].

A fact people cannot ignore when applying UAV-assisted SAR is the number of required operators. It is claimed that at least two roles are required: one pilot who flies, monitors, plans and controls the $\mathrm{UAV}$, and a second pilot who operates the sensors and information flow [14]. Practically, these two roles can be filled by a single operator, yet studies on ground robots have also suggested that a third person is recommended to monitor and protect the operator(s). Researchers have also studied the human behavior involved in managing multi UAVs, and have found that "the span of the human control is limited" $[4,14,15]$. As a result, a critical challenge of applying multiple UAVs in SAR is simultaneously monitoring information-rich data streams, including flight data and aerial video. The possibility of simplifying the human roles by optimizing information presentation and automatizing information acquisition was also explored [4], in which a fixed-wing UAV was used as a platform, and they analyzed and compared three computer vision algorithms to improve the presentation.

To automatize the information acquisition, it has been suggested that UAV systems integrate target-detection technologies for detecting people, cars or aircraft. A common method of observing people is the detection of heat features, which can be achieved by applying infrared camera technology and specifically developed algorithms. In 2005, a two-stage method based on a generalized template was presented [16]. In the first stage, a fast screening procedure is conducted to locate the potential person. Then, the hypothesized location of the person is examined by an ensemble classifier. In contrast, human detection based on color imagery has also been studied for many years. The research on developing a human detection method was conducted, which uses background subtraction, but pre-processing is required before a search mission [17]. Another method of human detection was presented that uses color images and models the human/flexible parts, then detects the parts separately [18]. A combination of both thermal and color imagery for human detection was also studied in [19].

To enhance information presentation and support humanitarian action, geo-referenced data from disaster-affected areas is expected to be produced. Numerous different technologies and algorithms for generating geo-referenced data via UAV have been studied and developed. A self-adaptive, image-matching technique to process UAV video in real-time for quick natural disaster response was presented in [20]. A prototype UAV and a geographical information system (GIS) by applying the stereo-matching method to construct a three-dimensional hazard map was also developed [21]. Scale Invariant Features Transform (SIFT) algorithms was improved in [22] by applying a simplified Forstner operator. Rectifying images on pseudo center points of auxiliary data were proposed in [23].

The aim of this study is to build an all-in-one camera-based target detection and positioning system that integrates the necessary remote sensors for wilderness SAR missions into a fixed-wing UAV. Identification and search algorithms were also developed. The UAV system can autonomously conduct a mission, including auto-takeoff and auto-landing. The on-board searching algorithm can report victims or cars with GPS coordinates in real-time. After the mission, a map of the hazard area can be generated to facilitate further logistics decisions and rescue troop action. Despite their importance, the algorithms for producing the hazard map are beyond the scope of this paper. In this work, we focus on the possibility of using a UAV to simultaneously collect geo-referenced data and detect victims. A hazard map and points are generated by the commercial software Pix4Dmapper ${ }^{\mathrm{TM}}$ (Pix4Dmapper Discovery version 2.0.83, Pix4D SA, Lausanne, Switzerland).

Figure 1 provides a mission flowchart. Once a wilderness SAR mission is requested to the Ground Control System (GCS), the GCS operator designs a flight path that covers the search area and 
sends the UAV into the air to conduct the mission. During the flight, the on-board image processing system is designed to identify targets such as cars or victims, and to report possible targets with the corresponding GPS coordinates to the GCS within $60 \mathrm{~m}$ accuracy. These real-time images and generalized GPS help the immediate rescue action including directing the victim to wait for rescue at the current location and delivering emergency medicine, food and water. Meanwhile, the UAV is transmitting real-time video to the GCS and recording high-resolution aerial video that can be used, once the UAV lands, in post-processing tasks such as target identification and mapping the affected area. The post-target identification is designed to report victims' accurate locations within $15 \mathrm{~m}$, and the map of the affected area can be used to construct a rescue plan.

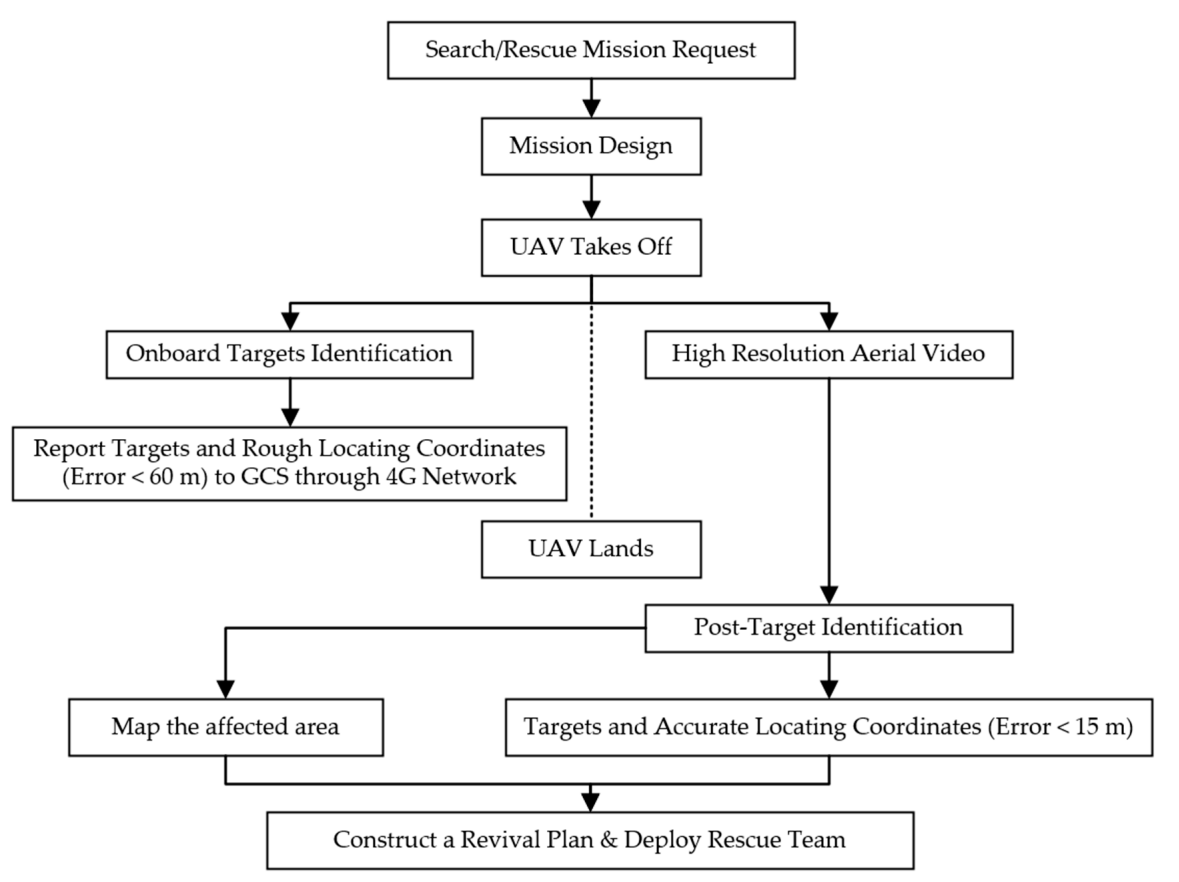

Figure 1. Flowchart of a wilderness SAR mission using the all-in-one UAV.

The remainder of this paper is organized as follows. Section 2 describes the details of the UAV system. Section 3 presents the algorithm and the implementation. Section 4 presents the tests and results, and Section 5 concludes the paper.

\section{Experimental Design}

The all-in-one camera-based target detection and positioning UAV system integrates the UAV platform, the communication system, the image system, and the GCS. The detailed hardware construction of the UAV is introduced in this section.

\subsection{System Architecture}

The purpose of the UAV system developed in this study was to find targets' GPS coordinates within a limited amount of time. To achieve this, a suitable type of aircraft frame was needed. The aircraft had to have enough fuselage space to accommodate the necessary payload for the task. The vehicle configuration and material had to exhibit the good aerodynamic performance and reliable structural strength needed for long-range missions. The propulsion system for the aircraft was calculated and selected once the UAV's configuration and requirements were known.

Next, a communication system, including a telemetry system, was used to connect the ground station to the UAV. After adding the flight control system, the aircraft could take off and follow the designed route autonomously. Finally, with the help of the mission system (auto antenna tracker (AAT), 
cameras, on-board processing board Odroid and gimbal), targets' and their GPS coordinates could be found. Figure 2 shows the UAV system's systematic framework, the details of which are explained in the following sub-sections. The whole system weighs $3.35 \mathrm{~kg}$ and takes off via hand launching.

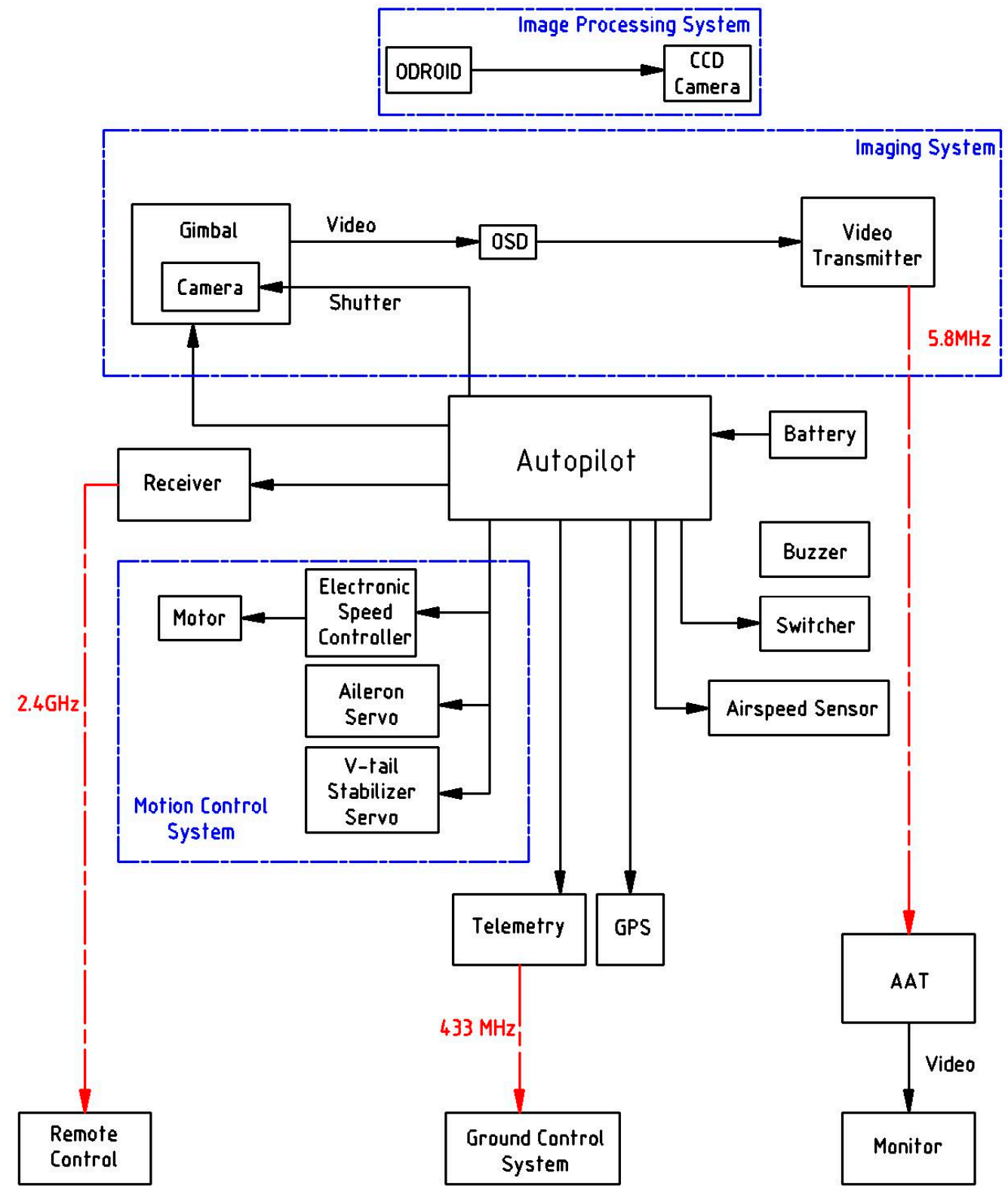

Figure 2. Systematic framework of the UAV system.

\subsection{Airframe of the UAV System}

The project objective was to develop a highly integrated system capable of large-area SAR missions. Thus, the flight vehicle, as the basic platform of the whole system, was chosen first. Given the prerequisites of quick response and immediate assessment capabilities, a fixed-wing aircraft was chosen for its high speed cruising ability, long range and flexibility in complex climatic conditions. To shorten the development cycle and improve system maintenance, an off-the-shelf commercial UAV platform "Talon" from X-UAV company was used (Figure 3). The wingspan of the Talon is $1718 \mathrm{~mm}$ and the wing area is $0.06 \mathrm{~m}^{2}$. The take-off weight of this airframe can reach $3.8 \mathrm{~kg}$. 


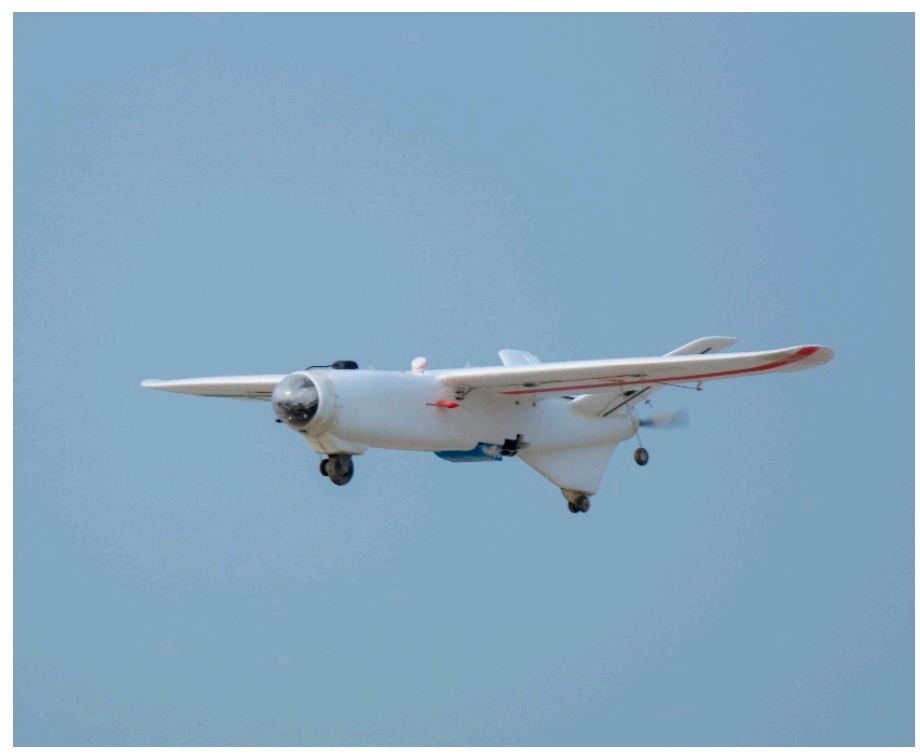

Figure 3. Overall View of X-UAV Talon [24].

\subsection{Propulsion System}

The UAV uses Sunnysky X-2820-5 motor works in conjunction with an APC 11X5.5EP propeller. A 10,000 $\mathrm{mAh}$ Lipo 4-cell $20 \mathrm{C}$ battery was used and this propulsion system provides a maximum cruse time of approximately $40 \mathrm{~min}$ at an airspeed of $18 \mathrm{~m} / \mathrm{s}$.

\subsection{Navigation System}

The main component of the navigation system is the Pixhawk flight controller running the free ArduPilot Plane firmware, equipped with GPS and compass kit, airspeed sensor and a sonar for measuring the height below $7 \mathrm{~m}$. The airplane with this navigation system can conduct a fully autonomous mission, including auto take-off, cruise via waypoints, return to home position and auto landing, with enhanced fail-safe protection.

\subsection{GCS and Data Link}

The GCS works via a data link that enables the researcher to monitor or interfere with the UAV during an auto mission. Mission Planner, an open-source ground station application compatible with Windows, was installed on the GCS laptop for mission design and monitoring. An HKPilot $433 \mathrm{Mhz}$ $500 \mathrm{Mw}$ radio transmitter and receiver was installed on the GCS laptop, along with a Pixhawk flight controller. An auto antenna tracker (AAT) worked in conjunction with a $9 \mathrm{dBi}$ patch antenna to provide a reliable data link within a 5-km range.

\subsection{Post-Imaging Processing and Video Transmission System}

The UAV system is designed with a fixed-wing aircraft flying at airspeeds ranging from 15 to $25 \mathrm{~m} / \mathrm{s}$ for quicker response times on SAR missions. The ground speed may reach $40 \mathrm{~m} / \mathrm{s}$ in extreme weather conditions. A GoPro HERO 4 was installed in the vehicle after considering the balance between its weight and image quality capabilities. In a searching and mapping mission, the aerial image always faces the ground. During flight, some actions such as rolling, pitching or other unexpected vibrations can disrupt the camera's stability, which may lead to unclear video. A Mini 2D camera gimbal produced by Feiyu Tech Co., Ltd. (Guilin, China), powered by two brushless motors, was used to stabilize the camera (Figure 4). The camera (GoPro HERO 4, GoPro, Inc., San Mateo, CA, USA) was set to video mode with a $1920 \times 1080$ pixel resolution in a narrow field of view (FOV) at 25 frames per second. During the flight, an analog image signal is sent to an on-screen display (OSD) 
and video transmitter. With a frequency of $5.8 \mathrm{GHz}$, the aerial video can be visualized by GCS in real-time as the high-resolution video is rerecorded for use during post-processing.

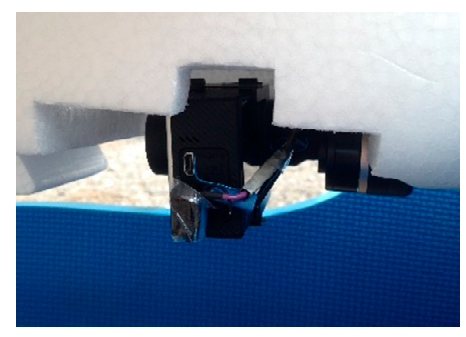

Figure 4. GoPro HERO 4 attached to the camera gimbal.

\subsection{On-Board, Real-Time Imaging Process and Transmission System}

A real-time imaging process and transmission system was setup on the UAV. The "oCam," (shows in Figure 5) a 5-mega pixel charge-coupled device (CCD) camera was chosen as the image source for the on-board target identification system. The focal length of the camera is $3.6 \mathrm{~mm}$ and it has a field of view of $65^{\circ}$. It weighs $37 \mathrm{~g}$ and has a $1920 \times 1080$ pixel resolution with 30 frames per second. The development of the on-board image processing was based on the Odroid XU4 (Hardkernel co., Ltd., GyeongGi, South Korea) (Figure 5b), which is a light, small, powerful computing device equipped with a 2-GHz core CPU and 2 Gbyte LPDDR3 Random-Access Memory (RAM). It also provides USB 3.0 interfaces that increase transfer speeds for high-resolution images. The Odroid XU4 used on the UAV in this system runs Ubuntu 14.04. The details of the algorithm and implementation will be discussed in Section 3. The Odroid board was connected to a 4th Generation (4G) cellular network via a HUAWEI (Shenzhen, China) E3372 USB dongle. Once the target is identified by the Odroid XU4, that particular image is transmitted through the 4G cellular network to the GCS.

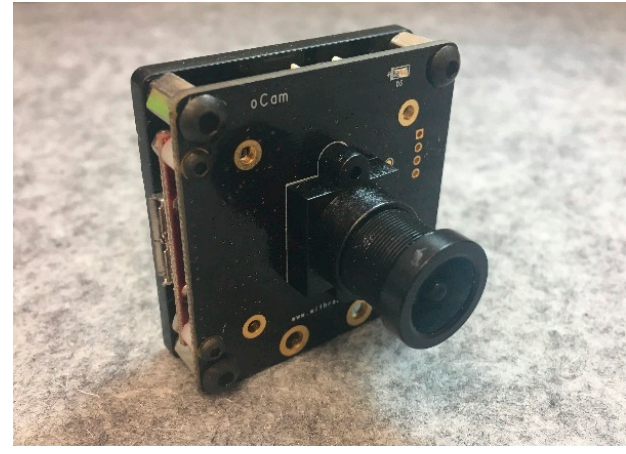

(a)

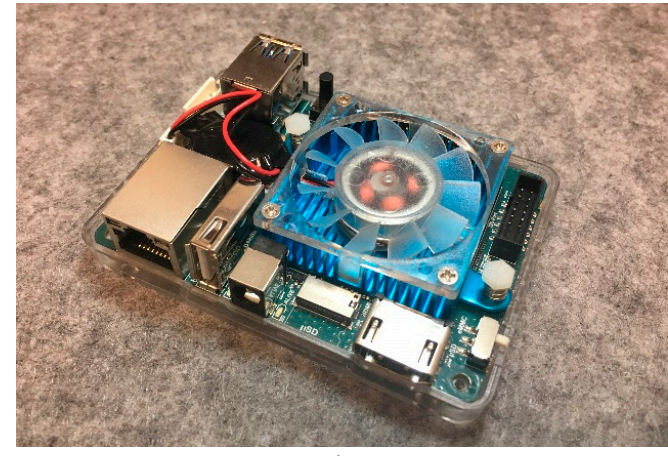

(b)

Figure 5. (a) oCam [25] and (b) Odroid XU4.

\section{Algorithm for and Implementation of Target Identification and Mapping}

The target identification program was implemented using an on-board micro-computer (Odroid XU4,) and the ground control station. The program can automatically identify and report cars, people and other specific targets.

\subsection{Target Identification Algorithm}

The mission is to find victims who need to be rescued, crashed cars or aircraft. The algorithm approaches these reconnaissance problems by using the color signature. These targets create a good contrast with the backgrounds due to their artificial colors. Figure 6 shows the flowchart of the reconnaissance algorithm. The aerial images are in YUV rather than RGB color space to identify the 
color signatures [26]. This progress can be achieved by calling back the function provided by OpenCV libraries. Both blue and red signatures are examined.

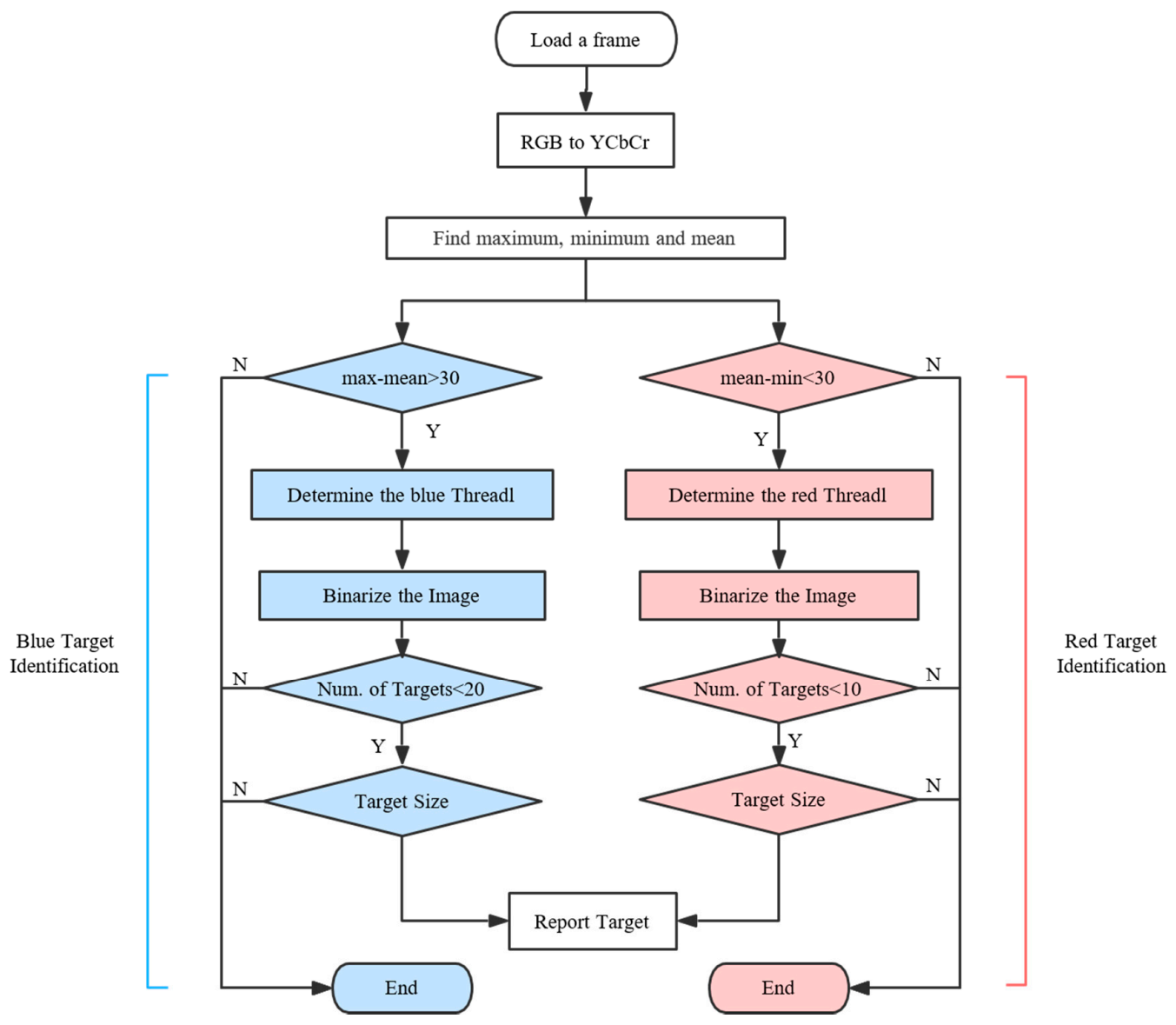

Figure 6. Flowchart of the identification algorithm.

The crucial step of the algorithm is to find an appropriate value of Threadl. A self-adapting method was applied to the reconnaissance program. The identification included the following steps.

Step 1: Read the blue and red chrominance values $(\mathrm{Cb}$ and $\mathrm{Cr}$ layers) of the image, and determine the maximum, minimum and mean values of the chrominance matrix. These values are then used to adapt the threshold.

Step 2: Distinguish whether existing objects are in great contrast. The distinction is processed by comparing the maximum/minimum and mean values of the chrominance. Introducing this step improves the efficiency with which the aerial video is processed, because the relevant identification is skipped if the criteria are not met. The criteria are expressed in Equation (1):

$$
\begin{aligned}
& \max -\text { mean }>30 \\
& \text { mean }-\min <30
\end{aligned}
$$

Step 3: Determine the appropriate value of the threshold, which is determined by Equation (2), where the threshold with subscripts $\mathrm{b}$ and $\mathrm{r}$ donate blue and red, respectively. $\mathrm{K}_{s}$ is the sensitivity factor, and the program becomes more sensitive as it increases. $\mathrm{K}_{s}$ also changes with different cameras, and was set as 0.1 for the GoPro HERO 4 and 0.15 for the oCam in this study.

$$
\begin{aligned}
\text { Threadl }_{b} & =\text { max }-(\text { max }- \text { mean }) * \mathrm{~K}_{s} \\
\text { Threadl }_{r} & =(\text { mean }- \text { min }) * \mathrm{~K}_{s}+\text { min }
\end{aligned}
$$


Step 4: Binarize the image with the threshold.

$$
f(p)=\left\{\begin{array}{r}
0 ;(p<\text { Threadl }) \\
255 ;(p>\text { Threadl })
\end{array}\right.
$$

where 0 represents the black color and 255 represents the white color.

Step 5: Examine the number of targets and their sizes. The results are abandoned if there are too many targets (over 20) in a single image because such results are typically caused by noise at the flight height of $80 \mathrm{~m}$. The amount criterion is used because it is rare for a UAV to capture over 20 victims or cars in a single image in the wilderness. When examining the size of the targets, the results are abandoned if the suspected target only has a few or too many pixels. The criterion for the number of pixels is determined by the height of the UAV and the size of the target.

Step 6: The targets are marked with blue or red circles on the original image and reported to the GCS.

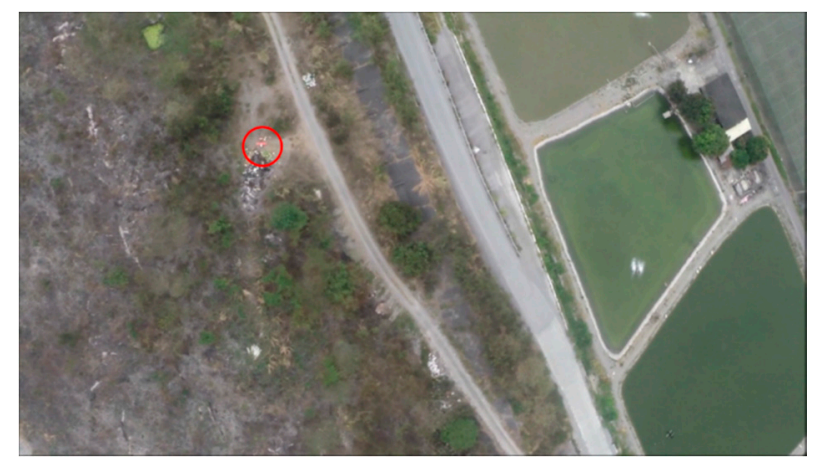

(a)

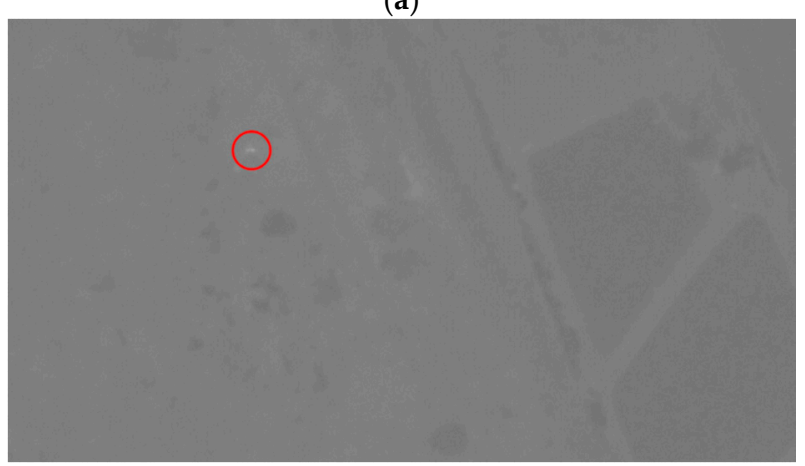

(b)

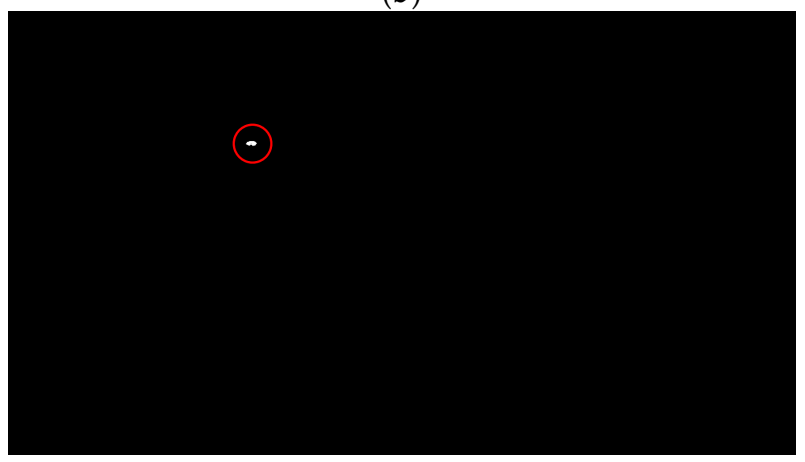

(c)

Figure 7. (a) The original image with red target in RGB color space; (b) the Cr layer of the YCbCr color space and (c) the binarized image with threshold. 
Figure 7 demonstrates a test of the target identification algorithm using an aerial image with a tiny red target. Figure 7a is the original image captured from the aerial video with the target circled for easy identification. The $\mathrm{Cr}$ data were loaded for red color, as shown in Figure 7b. Figure 7c shows the results of the binarized image with a threshold of 0.44 (the white spot in the upper left quadrant).

\subsection{On-Board Target Identification Implementation}

Before developing the on-board system for identifying targets, the method used to report the targets and their locations to the GCS must be determined. Considering all of the subsystems on the vehicle and the frequencies used for the data link (433 MHz), live video transmission $(5.8 \mathrm{GHz})$ and remote controller $(2.4 \mathrm{GHz})$, the on-board target identification system is designed to connect to the base station of a cellular network, $800-900 \mathrm{MHz}$ in the proposed testing area (Hong Kong and Taiwan). The results are then uploaded to the Dropbox server. Consequently, the on-board target identification system consists of four modules: Odroid as the core hardware, an oCam CCD camera, a GPS module and a dongle that connects to the $4 \mathrm{G}$ cellular network and provides it for the Odroid. The workflow of the on-board target identification system, designed as shown in Figure 8, includes three functions: Self-starting, identification and target reporting.

The self-starting is achieved via a Linux shell script. The program runs automatically when Odroid is powered on. The statuses of the camera, the Internet and the GPS module are checked. After successfully connecting all of the modules, the identification program runs on a loop until the Odroid is powered off. The identification program usually conducts four frames in a second.

During the flight, the GPS coordinates of the aircraft are directly treated as the location of the targets, because the rapid report is preferable to taking the time to get a highly accurate report during flight. The accurate locations of the targets are discovered post-flight using the high-resolution aerial video taken by the GoPro camera.

When reporting, the system scans the resulting files every $30 \mathrm{~s}$ and packs the new results, which are uploaded as a package instead of as frames to limit time consumption, because the Dropbox server requires verification for each file. The testing results show that uploading a package every $30 \mathrm{~s}$ is faster than uploading frame by frame. The reporting results include the images of the marked target and a text file of the GPS coordinates. These files are then stored in an external SD card that allows the GCS to quickly check the results post-flight. Figure 9 shows a truck reported by the on-board target identification system.

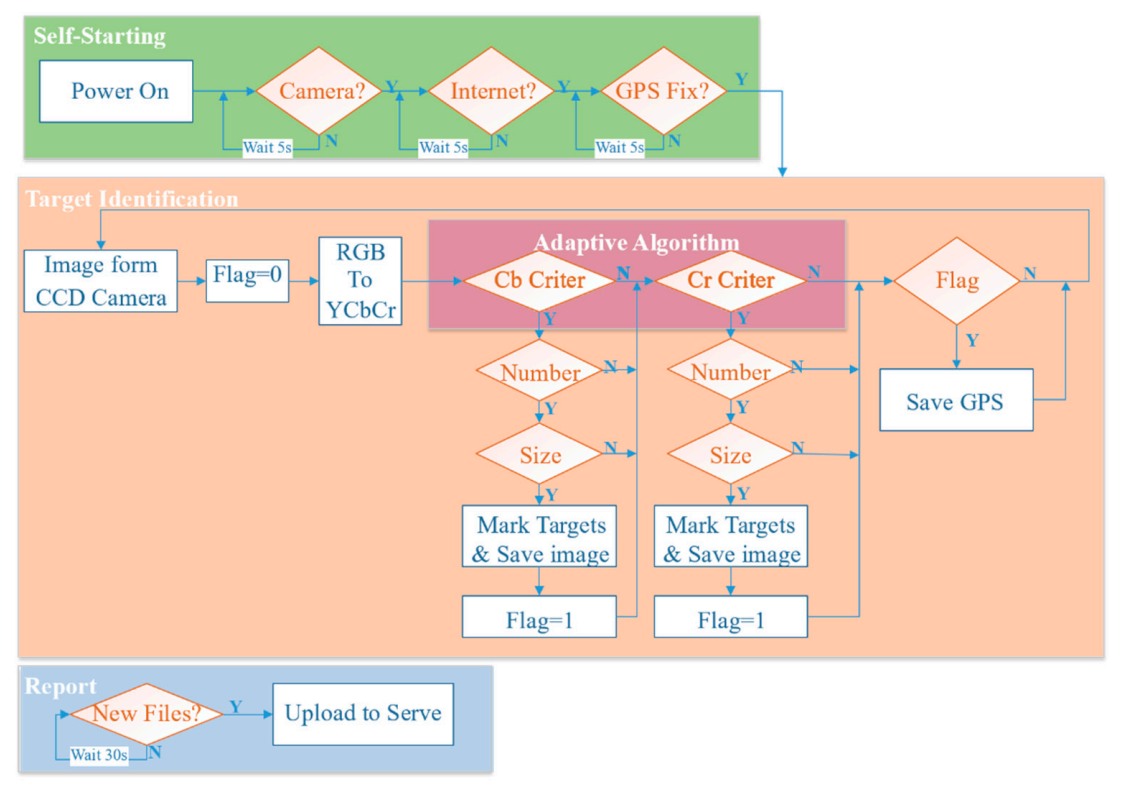

Figure 8. Flowchart of the on-board target identification system. 


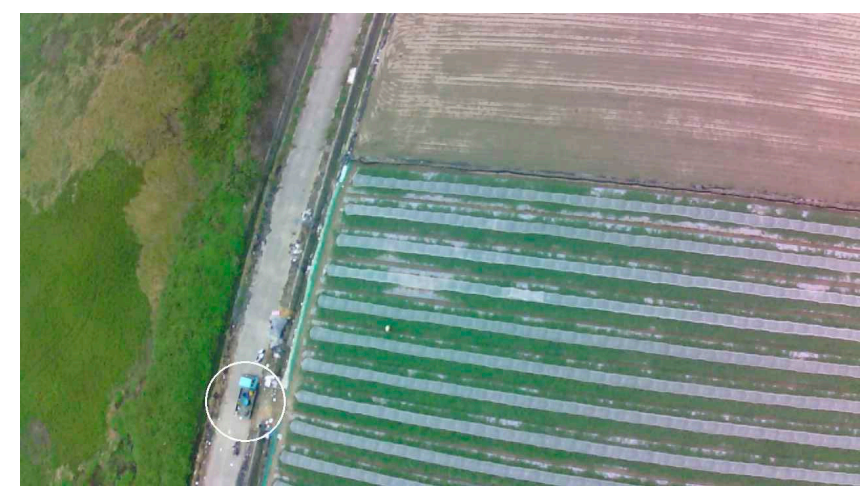

Figure 9. A blue truck reported by the on-board target identification system, marked by the identification program with a white circle.

\subsection{Post-Target Identification Implementation via Aerial Video and Flight Log}

Post-target identification is conducted using the high-resolution aerial video taken by the GoPro camera and stored in the SD card, and the flight data log from the flight controller to capture all possible targets to be rescued and obtain their accurate locations. In this section, the technical details of post-target identification are discussed.

\subsubsection{Target Identification}

The altitude of the flight path is carefully determined during the flight tests via the inertial-measurement unit and GPS data in the flight controller. Any targets coated with artificial colors of or larger than the estimated image size $(15 \times 15$ pixels), calculated according to the height of the UAV and the target's physical dimensions, should be reported.

Figure 10 shows an aerial image of a $0.8 \mathrm{~m} \times 0.8 \mathrm{~m}$ blue board with a letter ' $\mathrm{Y}$ ' on it from flight heights of $50 \mathrm{~m}, 80 \mathrm{~m}$ and $100 \mathrm{~m}$. The height of the flight path for the later field test was determined to be lower than $80 \mathrm{~m}$ accordingly, otherwise, the targets would only be several pixels in the image and might be treated as noise.

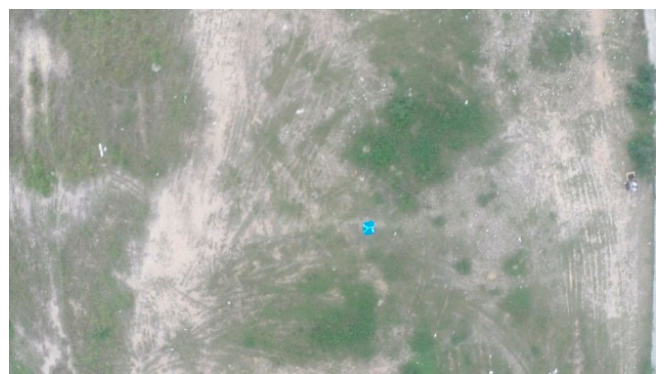

(a)

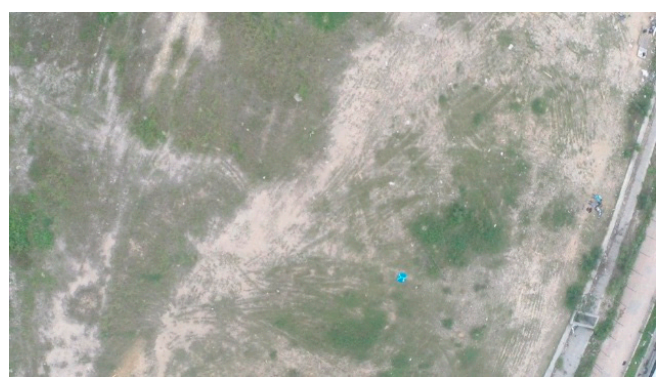

(b)

Figure 10. Cont. 


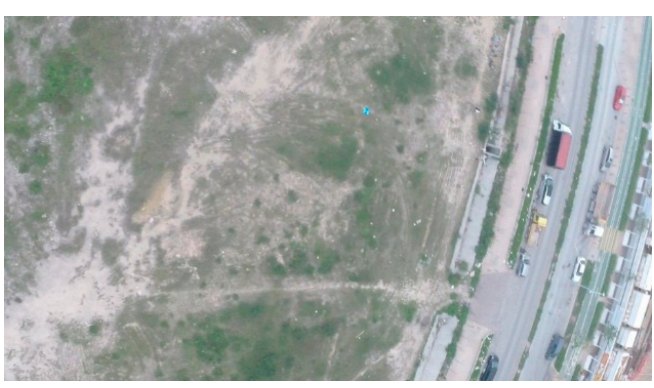

(c)

Figure 10. The results of altitude tests with the vehicle cruising at (a) $50 \mathrm{~m}$; (b) $80 \mathrm{~m}$ and (c) $100 \mathrm{~m}$.

The main loop of the post-identification program was developed in the OPENCV environment. Similar to on-board target identification, the post-identification program loads the aerial video file and runs the algorithm in a loop with each frame. The targets are marked for the GCS operator, who engages in efficient confirmation. The flight data log and the aerial video are simultaneously synchronized to determine the reference frame number and reference shutter time. The technical details of this step are discussed in Section 3.3.2. The target image is saved as a JPEG file and named with its frame number. Figure 11 shows a red target board and a green agricultural net reported by the post-identification program. This JPEG file is sent to the GPS transformation program discussed in Section 3.3.3 to better position the target.

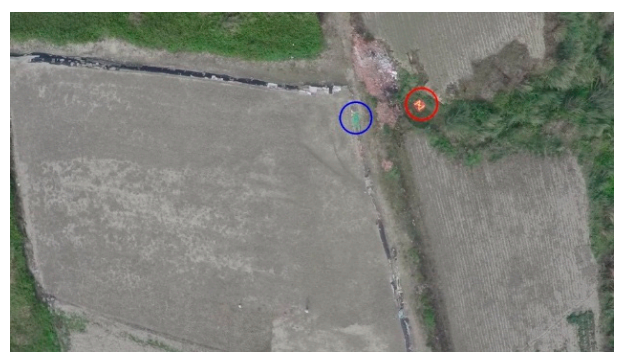

Figure 11. A red target board and a green agricultural net reported by the post-identification program, with both the red and blue targets marked with circles in corresponding colors.

To determine the image's frame number, we assume that the GoPro HERO 4 camera records the video with a fixed frame rate of 25 frames per second (FPS) in this study. Thus, the time interval (TI) of the target frame $\mathbf{F}$ in the aerial video and the reference frame can be determined by

$$
T I=(\text { Frame Number }- \text { Reference Frame No. }) \times 40 \mathrm{~ms}
$$

and the GPS time of $\mathbf{F}$ is

$$
\text { GPSTime }=\text { Reference GPS Time }+ \text { TI }
$$

where the Reference Frame No. and Reference GPS Time are determined during synchronization, as discussed in Section 3.3.2.

Once the GPS time of the target frame is determined, the altitude and GPS coordinates of the camera are determined. The yaw angle $\Psi$ is recorded as part of the Attitude messages in the flight data $\log$, and the corresponding Attitude message can be searched via GPS time. The update frequencies of the Attitude messages come from an inertial-measurement unit IMU sensor, and the GPS messages are different. These two types of messages cannot be recorded simultaneously due to the control logic of the flight board. However, the updating frequency of the Attitude message is much higher than that of the GPS messages, thus the attitude message that is closest to the GPS time is treated as the vehicle's current attitude. 


\subsubsection{Synchronization of the Flight Data and Aerial Video}

During the flight, the aerial video and flight data are recorded by the GoPro HERO 4 camera and flight controller, respectively. It is crucial to synchronize the flight data and the aerial video to obtain the targets' geo-information for the identification and mapping of the affected areas in a rescue mission.

Camera trigger distance (DO_SET_CAM_TRIGG_DIST), a camera control command provided by ArduPlane firmware, was introduced to synchronize the aerial video and the flight data log. DO_SET_CAM_TRIGG_DIST sets the distance in meters between camera triggers, and the flight control board logs the camera messages, including GPS time, GPS location and aircraft altitude when the camera is triggered. Compared with commercial quad-copters, fixed-wing UAVs fly at higher airspeeds. The time interval between two consecutive images should be small enough to meet the overlapping requirement for further mapping. However, the normal GoPro HERO 4 cannot achieve continuous photo capturing at a high frequency $(5 \mathrm{~Hz}$ or $10 \mathrm{~Hz})$ for longer than $30 \mathrm{~s}$ [27]. Thus, the GoPro was set to work in video recording mode with a frame rate of 25 FPS. The mode and shutter buttons were modified with a pulse width modulation (PWM)-controlled relay switch, as shown in Figure 12, so that the camera can be controlled by the flight controller. The shutter and its duration are configured in the flight controller.

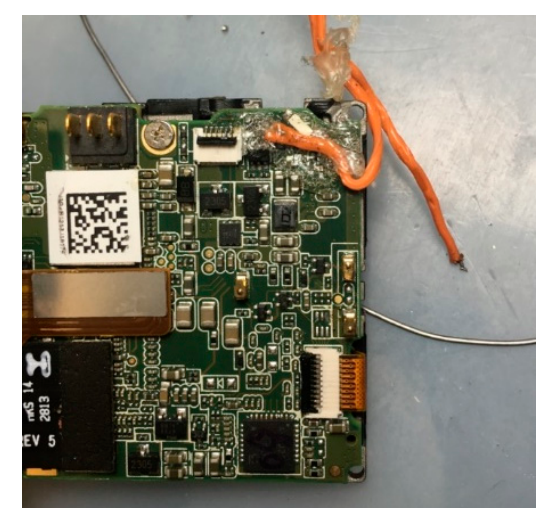

Figure 12. Modification of the GoPro buttons to PWM-controlled relay switch.

The camera trigger distance can be set to any distance that will not affect the GoPro's video recording. A high-frequency photo capturing command will lead to video file damage. In this study, the flight controller sends a PWM signal to trigger the camera and record the shutter times and positions of the camera messages. However, the Pixhawk records the time that the control signal is sent out, and there is a delay between the image's recorded time and its real shutter time. This shutter delay was measured to be $40 \mathrm{~ms}$ and was introduced to the synchronization process.

The synchronization process shown in Figure 13 is conducted after the flight. The synchronization process shown in Figure 13 is conducted after the flight. The comparison process started with reading the aerial video and the photograph saved in GoPro's SD card. The original captured photo was resized to $1920 \times 1080$ pixels because the GoPro photograph was of a nonstandard size of $2016 \times 1128$ pixels. During the comparison process, both the video frames and photograph were treated as a matrix with a size of $1920 \times 1080 \times 3$, where the number 3 denotes the 3 layers of RGB color space. The difference $\varepsilon$ between the video frame and the photo was determined by the mean-square deviation value of $\left(\right.$ Matrix $_{\text {photo }}-$ Matrix $\left._{\text {frame }}\right)$. The video frame with minimum value of $\varepsilon$ was considered the same as the original aerial photo (Figure 14) and the number of this video frame was recorded as the Reference Frame No (RFN). The recorded GPS time of sending the aerial photo triggering command was named as the Reference GPS time (RGT). Considering the above-mentioned $40 \mathrm{~ms}$ delay between sending out the command and capturing the photo the frame at RFN was taken at the time of (RGT + 40 ms delay time). Therefore, the video is combined with the flight log. 


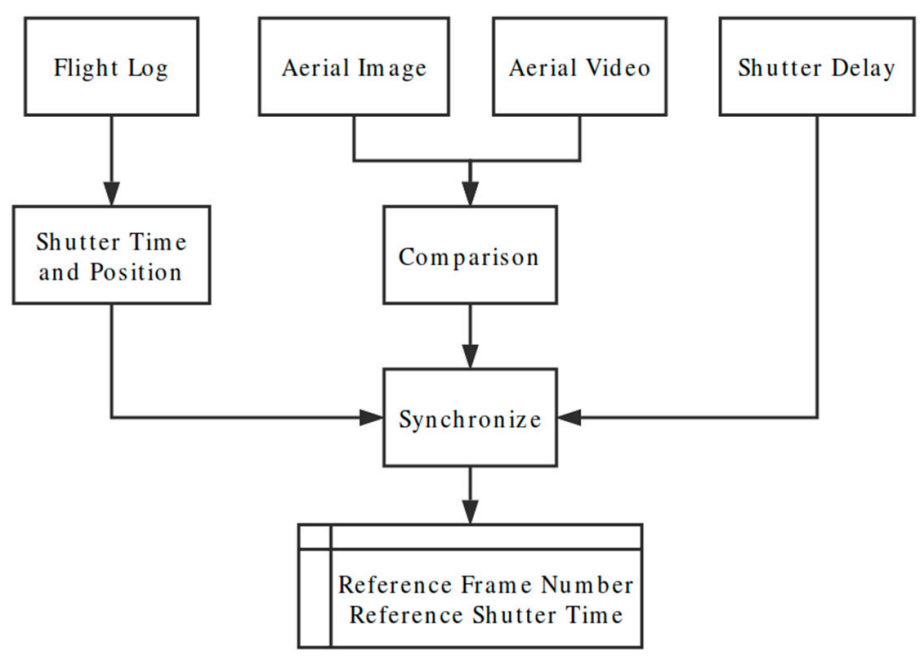

Figure 13. Flowchart for the synchronization of the aerial video and the flight data log.

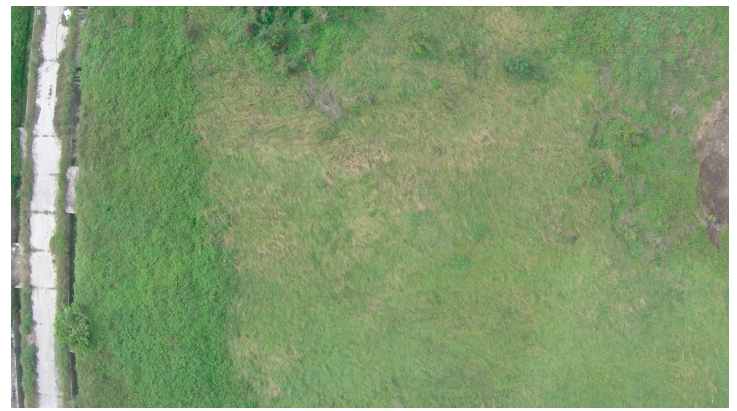

(a)

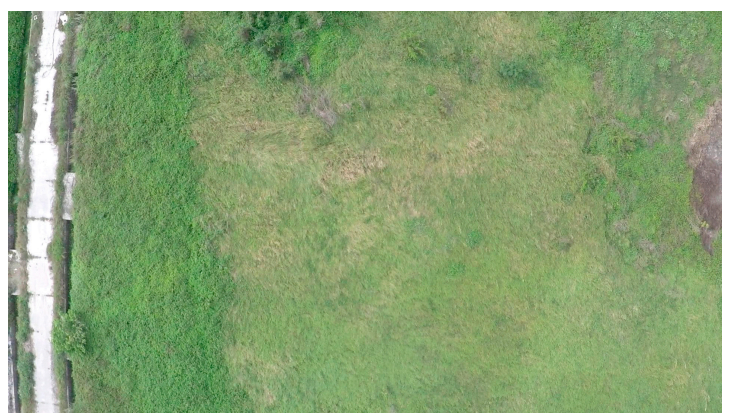

(b)

Figure 14. Comparison results in synchronization process (a) the original photo taken by Gopro camera and (b) video frame captured by synchronization program.

\subsubsection{GPS Transformation to Locate Targets}

Once a target with its current aircraft position is reported to the GCS, an in-house MatLab locating program is used to report the target's GPS coordinates. In this study, the position of the aircraft is assumed to be at the center of the image, because the GPS module is placed above the camera.

The coverage of an image can be estimated using the camera's field of view (FOV) [28], as shown in Figure 15. The distances in the $\mathrm{x}$ and $\mathrm{y}$ directions are estimated using Equation (6).

$$
\begin{aligned}
& a=\frac{2 h}{\cos \left(\frac{\mathrm{FOV}}{2}\right)} \\
& b=\frac{2 h}{\cos \left(\frac{\mathrm{FOV}_{\mathrm{Y}}}{2}\right)}
\end{aligned}
$$

The resolution of the video frame is set to be $1920 \times 1080$ pixels. The scale between the distance and pixels is assumed to be a linear relationship, and is presented in Equation (7) as:

$$
\begin{aligned}
& \text { scale }_{x}=\frac{a}{1920}=\frac{2 h}{1920\left(\frac{\mathrm{FOV}}{2}\right)} \\
& \text { scale }_{y}=\frac{b}{1080}=\frac{2 h}{1080\left(\frac{\mathrm{FOV}}{2}\right)}
\end{aligned}
$$




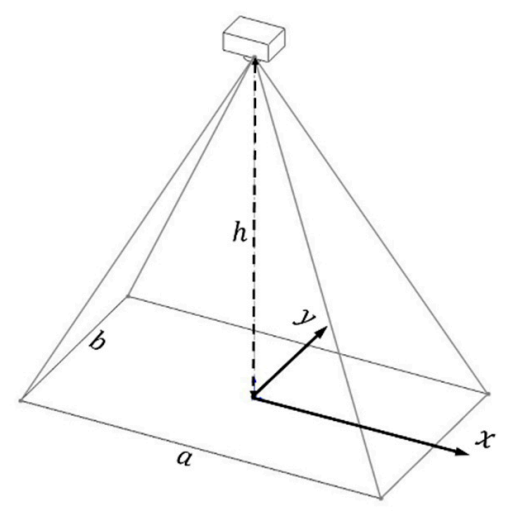

(a)

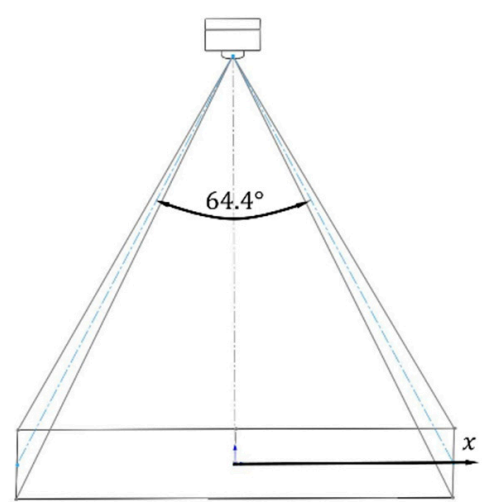

(b)

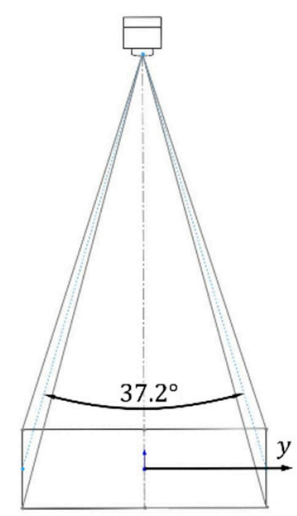

(c)

Figure 15. Camera and world coordinates.

As Figure 16 shows, a target is assumed to be located on the $(x, y)$ pixel in the photo, and the offset of the target from the center of the picture is

$$
\text { offset }_{\text {target }}=\left[\begin{array}{l}
\text { scale }_{x} \cdot x \\
\text { scale }_{y} \cdot y
\end{array}\right](\mathrm{m})
$$

For the transformation of a north-east (NE) world-to-camera frame with the angle of the $\Psi$, the rotation matrix is defined as

$$
\mathbf{R}_{\mathbf{W}}^{\mathbf{C}}=\left[\begin{array}{cc}
\cos (\Psi) & -\sin (\Psi) \\
\sin (\Psi) & \cos (\Psi)
\end{array}\right]
$$

where $\Psi$ is the yaw angel of the aircraft. Thus, the position offset in the world frame can be solved with

$$
\boldsymbol{P}=\mathbf{R}_{\mathbf{W}}^{\mathbf{C}^{\mathrm{T}}} \text { offset }_{\text {target }}=\left[\begin{array}{c}
P_{E} \\
P_{N}
\end{array}\right]
$$

Therefore, the target's GPS coordinates can be determined using

$$
\boldsymbol{G P S}_{\text {target }}=\boldsymbol{G P S}_{\text {cam }}+\left[\begin{array}{c}
P_{E} / f_{x} \\
P_{N} / f_{y}
\end{array}\right]
$$

where $f_{x}$ and $f_{y}$ denote the distances represented by one degree of longitude and latitude, respectively.

A graphical user interface was designed and implemented in the MatLab environment to transform the coordinates with a simple 'click and run' function (Figure 17). The first step is opening the image containing the targets. The program automatically loads the necessary information for the image, including the frame number (also the image's file name), current location, camera attitude and yaw angle of the plane. The second step is to click the 'GET XY' button and use the mouse to click the target in the image. The program shows the coordinates of the target in this image. Finally, clicking the 'GET GPS' button provides the GPS coordinates reported by the program. 


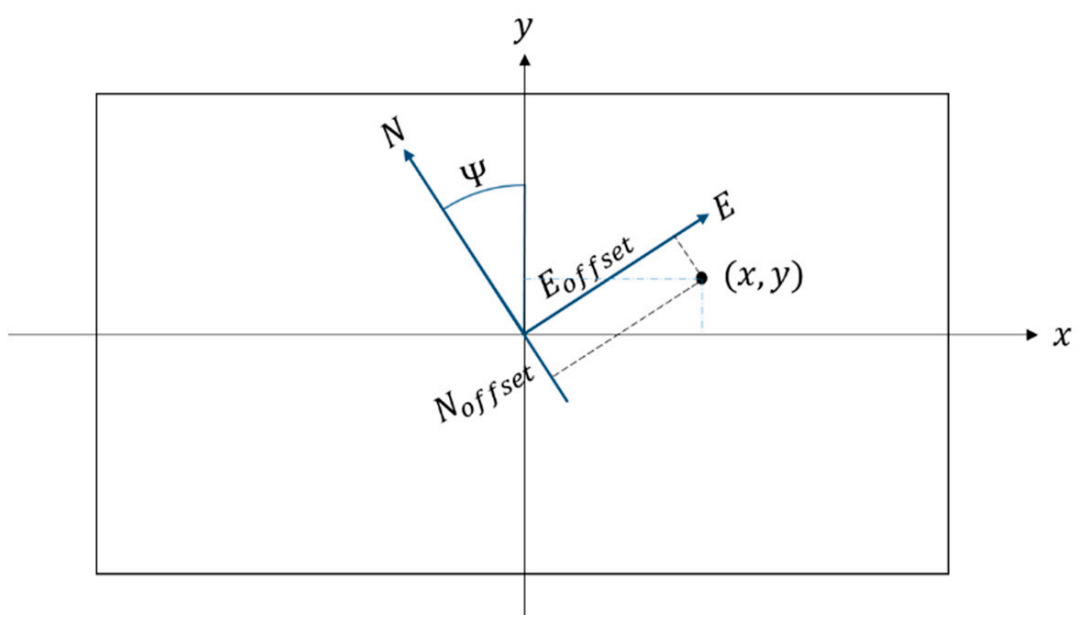

Figure 16. Coordinates of the camera and world frames.
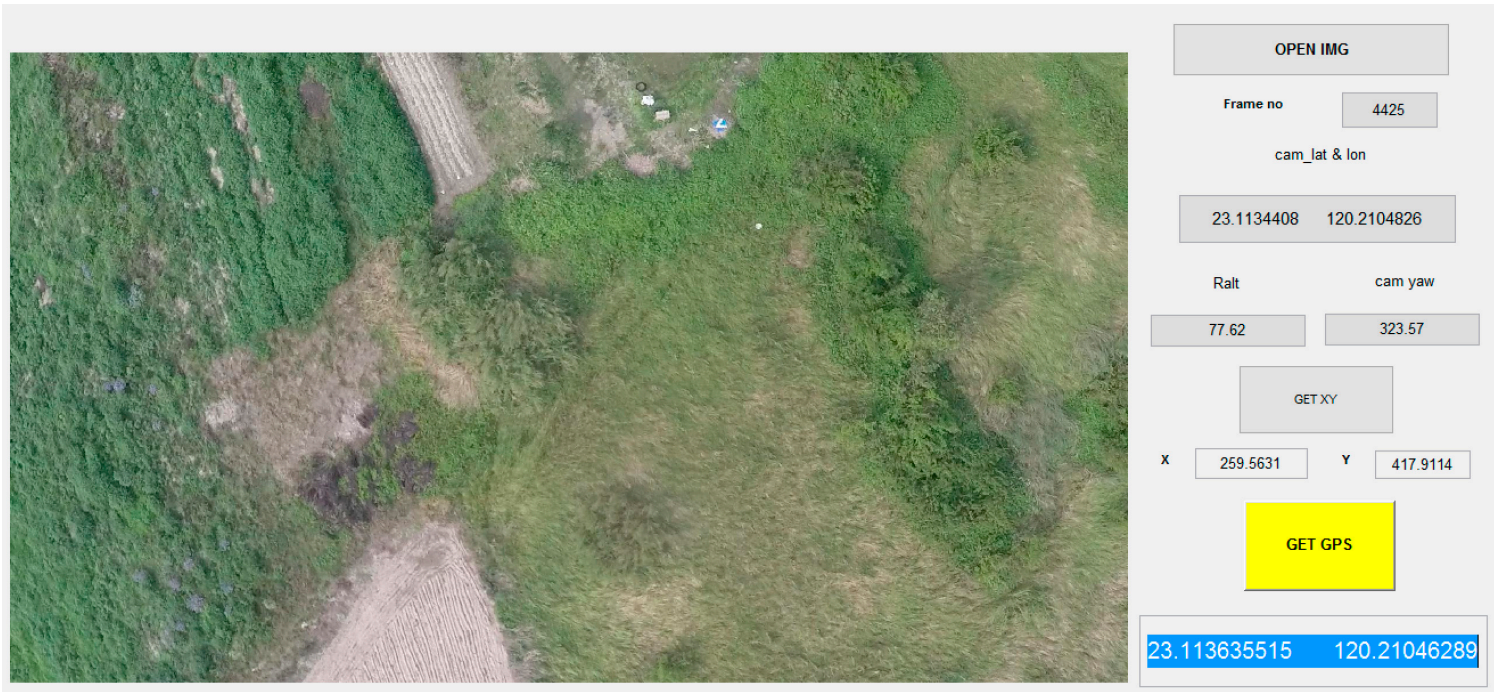

Figure 17. Graphical user interface for the GPS transformation that allows end users to access a target's GPS coordinates using simple buttons.

\subsection{Mapping the Searched Area}

During rescue missions following landslides or floods, the terrain features can change significantly. After target identification, the local map must be re-built to guarantee the rescue team's safety and shorten the rescue time. In this study, we provide a preliminary demonstration of a fixed-wing UAV used to assist in post-disaster surveillance. Mapping algorithms are not discussed in this paper. The commercial software Pix4D was used to generate orthomosaic models and point clouds.

To map the disaster area, a set of aerial photos and their geo-information are applied to the commercial software, Pix $4 \mathrm{D}$. There should be at least $65 \%$ overlap between consecutive pictures, but aiming for $80 \%$ or higher is recommended. The distance between two flight paths should be smaller than $a$, and estimation Equation (6) can be found in Section 3.3.3. A mapping image capture program is shown in Figure 18. 


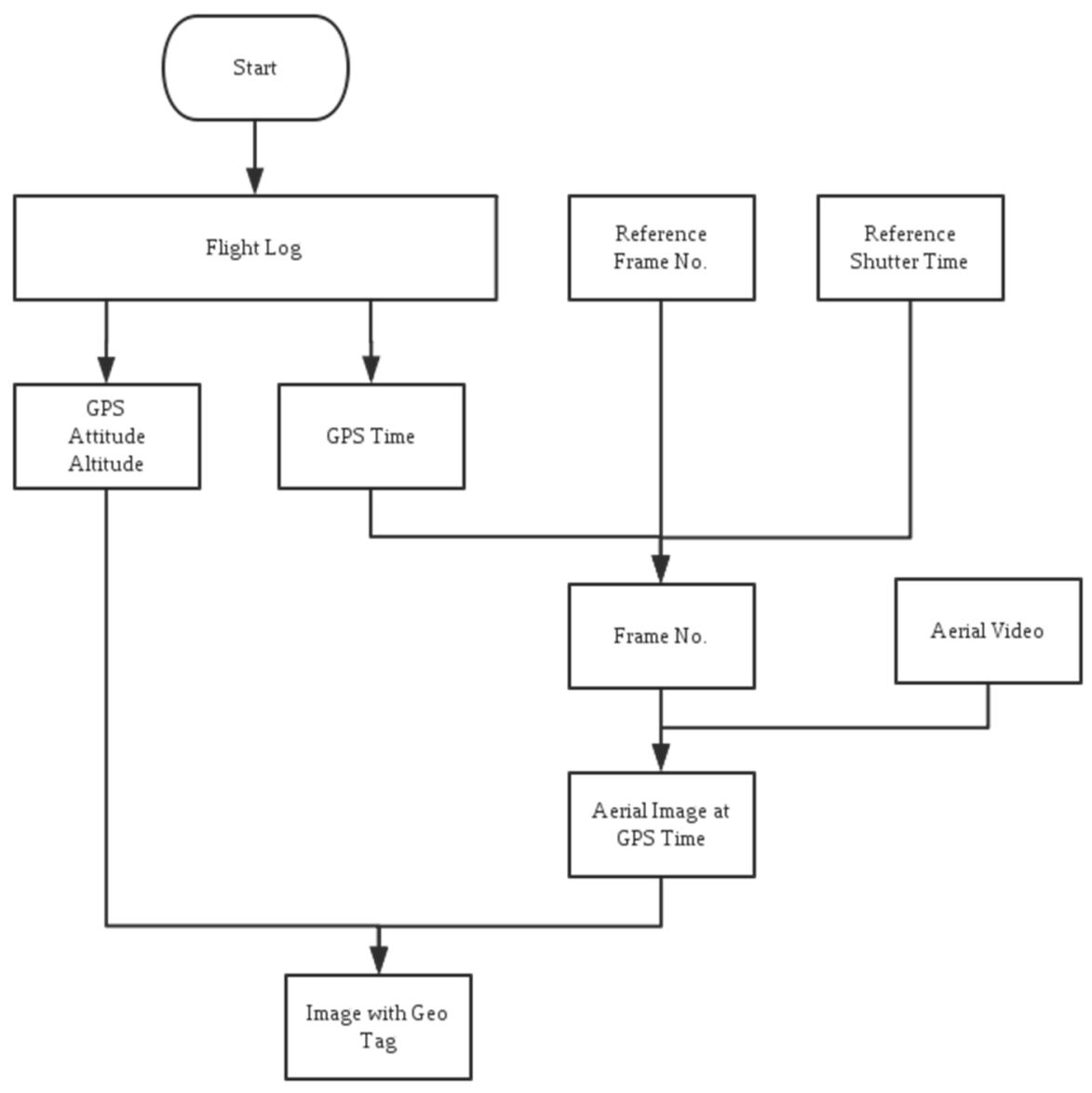

Figure 18. Flowchart of the mapping image capture program.

The mapping image capture program starts with GPS messages from the flight data log with reference frame numbers and shutter times generated by the synchronization step discussed in Section 3.2. The program loads the GPS times of all of the GPS messages in the loop and calculates the corresponding frame number $N$ in the aerial video, which equals

$$
N=\frac{\text { GPS Time }- \text { Reference GPS Time }}{40 \mathrm{~ms}}+\text { Reference Frame No. }
$$

Then, the mapping image capture program loads the Nth frame of the aerial video and saves it to the image file.

Once the mapping image capture program is complete, a series of photos and a text file containing the file names, longitude, latitude, altitude, roll, pitch and yaw are generated. The Pix4D then produces the orthomosaic model and point clouds using these two file types.

\section{Blind Tests and Results}

To test the all-in-one camera-based target detection and positioning system, a blind field test was designed. A drone, a $2 \mathrm{~m} \times 2 \mathrm{~m}$ blue or red square board and a $0.8 \mathrm{~m} \times 0.8 \mathrm{~m}$ blue or red square board were used to simulate a crashed airplane, broken cars and injured people, respectively (Figure 19a-c).

The flight tests were conducted at two test sites, the International Model Aviation Center $\left(22^{\circ} 24^{\prime} 58.1^{\prime \prime} \mathrm{N} 114^{\circ} 02^{\prime} 35.4^{\prime \prime} \mathrm{E}\right)$ of the Hong Kong Model Engineering Club, Ltd. in Yuen Long town, Hong Kong and the Zengwun River $\left(23^{\circ} 7^{\prime} 18.03^{\prime \prime} \mathrm{N} 120^{\circ} 13^{\prime} 53.86^{\prime \prime} \mathrm{E}\right)$ in the Xigang District, of Tainan city, Taiwan. Given concerns with the limited flying area in Hong Kong, the preliminary in-sight tests were conducted in Hong Kong and the main blind out-of-sight tests were conducted in 
Taiwan. The flight test information is listed in Table 1. Only post-identification tests were conducted in Hong Kong. In Taiwan, no after-flight mapping was done for the first two tests (Tests 3 and 4).

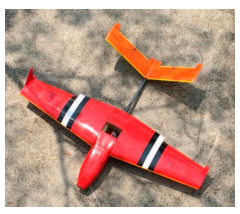

(a)

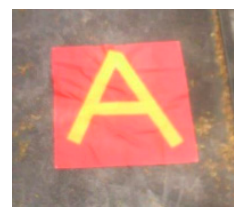

(b)

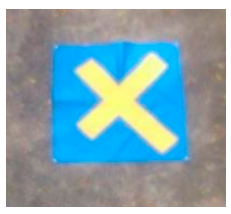

(c)

Figure 19. (a) The drone simulated a crashed airplane, (b) the $2 \mathrm{~m} \times 2 \mathrm{~m}$ blue or red target boards represented broken cars and $(\mathrm{c})$ the $0.8 \mathrm{~m} \times 0.8 \mathrm{~m}$ blue or red targets boards represented injured people to be rescued.

Table 1. Basic information for flight tests.

\begin{tabular}{cccccc}
\hline \multirow{2}{*}{ Flight Test } & Test Site & Flight Time (min) & $\begin{array}{c}\text { Real-Time } \\
\text { Identification }\end{array}$ & Post-Identification & Mapping \\
\hline Test 1 & Hong Kong & $15: 36$ & $\times$ & $\sqrt{ }$ & $\times$ \\
Test 2 & Hong Kong & $3: 05$ & $\times$ & $\sqrt{ }$ & $\times$ \\
Test 3 & Taiwan & $13: 23$ & $\sqrt{ }$ & $\sqrt{ }$ & $\times$ \\
Test 4 & Taiwan & $17: 41$ & $\sqrt{ }$ & $\sqrt{ }$ & $\sqrt{ }$ \\
Test 5 & Taiwan & $17: 26$ & $\sqrt{ }$ & $\sqrt{ }$ & $\sqrt{ }$ \\
Test 6 & Taiwan & $16: 08$ & $\sqrt{ }$ & $\sqrt{ }$ & $\sqrt{ }$ \\
Test 7 & Taiwan & $16: 23$ & $\sqrt{ }$ & & \\
Test 8 & Taiwan & $17: 56$ & &
\end{tabular}

Figure 20a,b shows the search site and its schematic in Hong Kong. The search path repeated the square route due to the limited flight area. The yellow path in Figure 20a is the designed mission path and the purple line indicates the real flight path of the vehicle. For the tests in Taiwan, there were two main search areas (A and B) along the bank of the Zengwun River in the Xigang District of Tainan city, Taiwan, as shown in Figure 20c. The schematics of the designed search route and areas are depicted in Figure 20d. The maximum communication distance was $3 \mathrm{~km}$ and the width of the flight corridor was $30 \mathrm{~m}$. This width was intended to test the stability of the UAV and the geo-fencing function of the flight controller. If the UAV flies outside the corridor, it is considered to have crashed. After the flight performance tests, the UAV flew inside the corridor and was proven stable. An unknown number of targets were placed in search areas A and B by an independent volunteer before every test. The search team then conducted the field tests and tried to find the targets. The test results are discussed in the following sections.

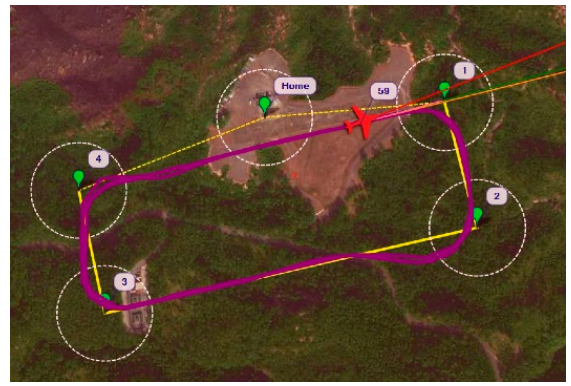

(a)

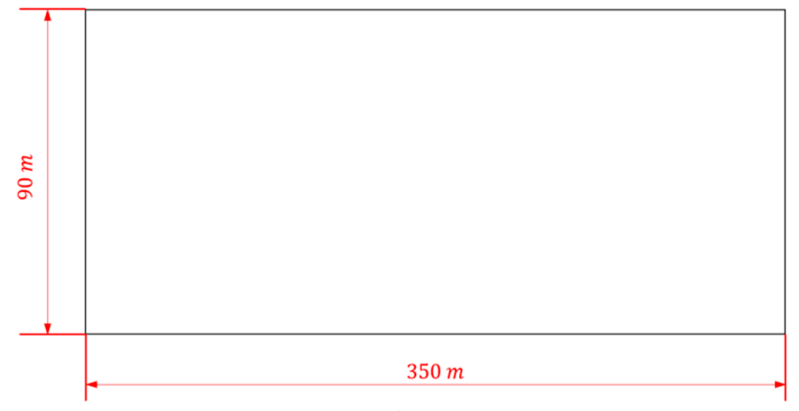

(b)

Figure 20. Cont. 


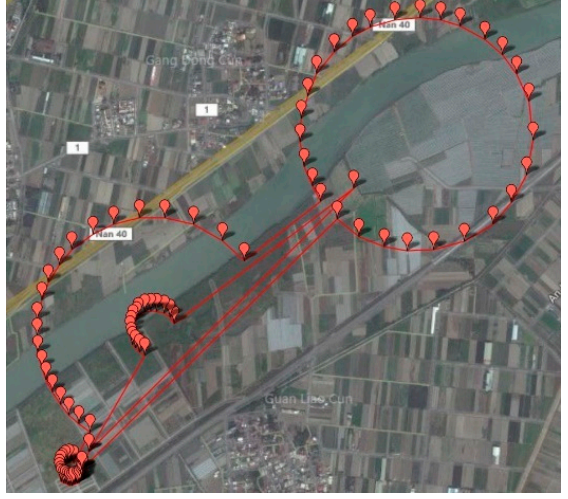

(c)

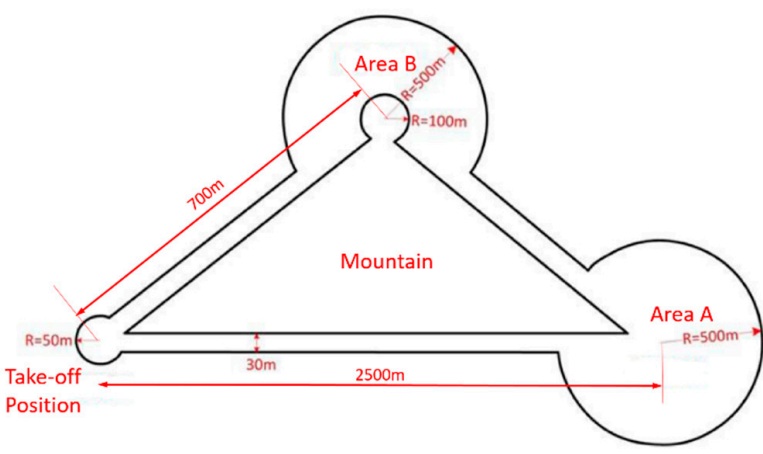

(d)

Figure 20. (a) Test route in Hong Kong; (b) schematics of the designed route in Hong Kong; (c) search areas A and B for blind tests in Taiwan and (d) schematics of the designed search route and areas in Taiwan.

\subsection{Target Identification and Location}

Post-target identification processing was conducted in all eight flight test to assess the identification algorithm. The post-identification program ran on a laptop equipped with Intel Core i5-2430M CPU and 8 Gb RAM. The testing results are shown in Table 2. Note that the post-identification program only missed two targets for all of the tests.

Table 2. Post-target identification results.

\begin{tabular}{|c|c|c|c|c|c|c|}
\hline Flight Test & Resolution & Flying Altitude & Flight Time (min) & Targets & Identified Targets & $\begin{array}{l}\text { Total Post-Target } \\
\text { Identification } \\
\text { Time (min) }\end{array}$ \\
\hline Test 1 & $1920 \times 1080$ & 80 & $15: 36$ & 3 & 2 & 11:08.6 \\
\hline Test 2 & $1920 \times 1080$ & 80 & 3:05 & 2 & 2 & $02: 46.3$ \\
\hline Test 3 & $1920 \times 1080$ & 80 & $13: 23$ & 3 & 3 & $12: 57.1$ \\
\hline Test 4 & $1920 \times 1080$ & 80 & $17: 41$ & 3 & 2 & $14: 04.6$ \\
\hline Test 5 & $1920 \times 1080$ & 80 & $17: 26$ & 3 & 3 & $13: 23.9$ \\
\hline Test 6 & $1920 \times 1080$ & 80 & $16: 08$ & 3 & 3 & $11: 45.1$ \\
\hline Test 7 & $1920 \times 1080$ & 80 & $16: 23$ & 6 & 6 & $12: 16.9$ \\
\hline Test 8 & $1920 \times 1080$ & 75 & $17: 56$ & 6 & 6 & $14: 18.3$ \\
\hline
\end{tabular}

Taking test 7 as an example, $6 / 6$ targets were found by the identification system, as shown in Figure 21, including a crashed aircraft, two crashed cars and three injured people. Note that in Figure 21g the target board, representing the injured people, was folded by gusts of wind to the extent that it is barely recognizable. Nevertheless, the identification system still reported this target, confirming its reliability. The locating error of 5 targets was less than $15 \mathrm{~m}$ as shown in Table 3 (having met the requirements discussed in Section 1). The targets and their locations were reported in $15 \mathrm{~min}$.

Table 3. Locating results of flight test 7.

\begin{tabular}{ccccccc}
\hline Target & Red Z & Red Plane & Blue I & Blue V & Blue J & Red Q \\
\hline Latitude (N) & $23.114536^{\circ}$ & $23.111577^{\circ}$ & $23.110889^{\circ}$ & $23.113637^{\circ}$ & $23.122189^{\circ}$ & $23.117840^{\circ}$ \\
Longitude (E) & $120.213111^{\circ}$ & $120.211898^{\circ}$ & $120.210819^{\circ}$ & $120.210463^{\circ}$ & $120.223206^{\circ}$ & $120.225225^{\circ}$ \\
Error & $2.8 \mathrm{~m}$ & $13.9 \mathrm{~m}$ & $1.6 \mathrm{~m}$ & $0.8 \mathrm{~m}$ & $11.3 \mathrm{~m}$ & $4.8 \mathrm{~m}$ \\
\hline
\end{tabular}




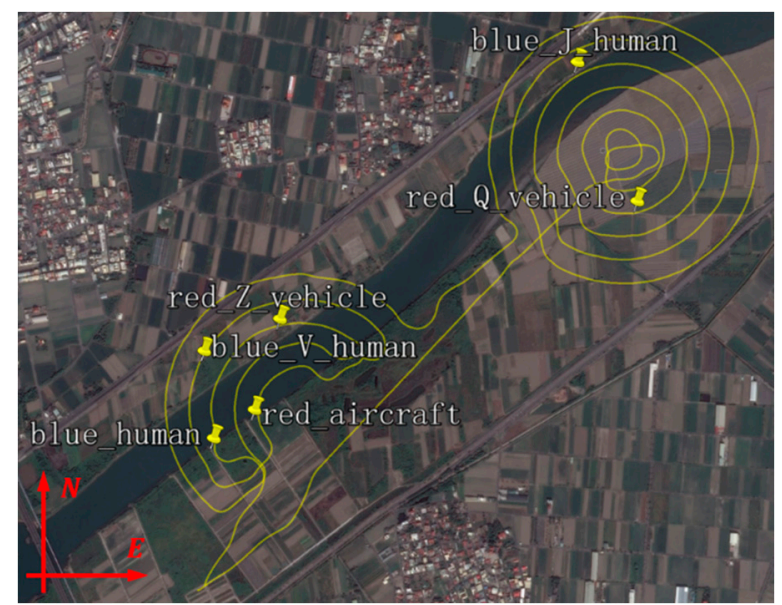

(a)

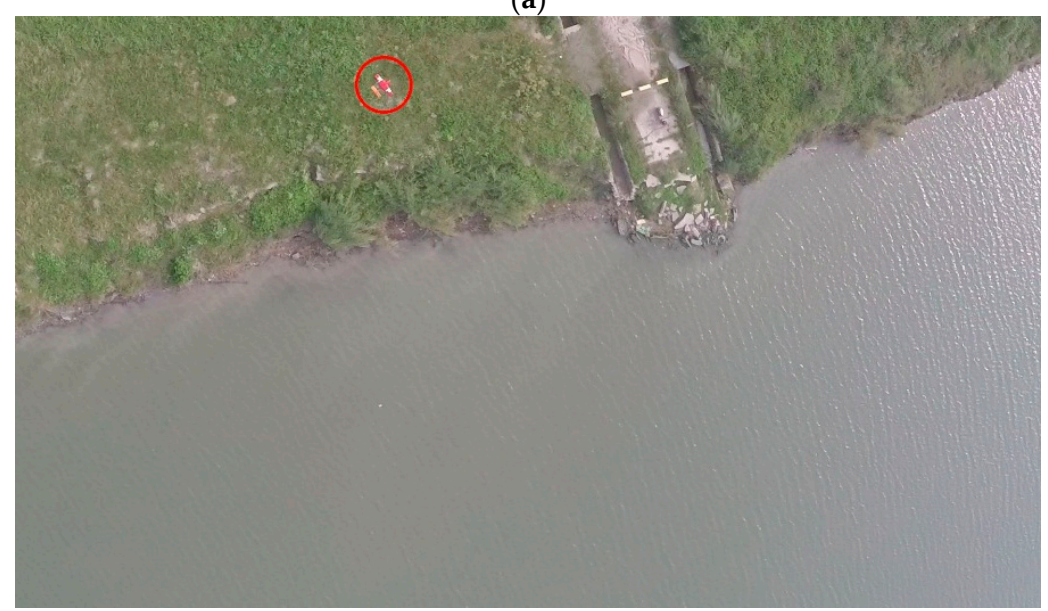

(b)

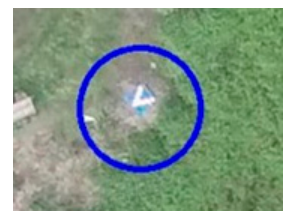

(c)

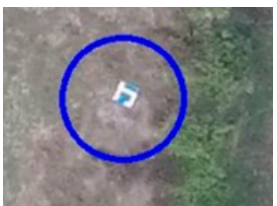

(d)

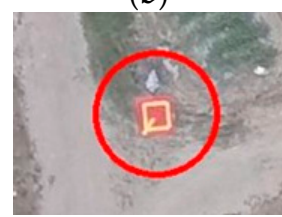

(e)

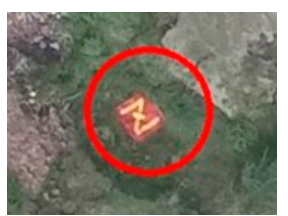

(f)

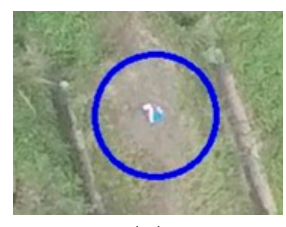

(g)

Figure 21. (a) The locations of six simulated targets; (b) the original image saved by the identification program with target drone; (c) designed target (blue board with letter V) represents an injured person; (d) designed target (blue board with letter J) represents an injured person; (e) designed target (red board with letter $Q$ ) represents a crashed car; (f) designed target (red board with letter Z) represents a crashed car and (g) designed target (small blue board) represents an injured person. The board was blown over by the wind.

In addition to the designed targets, the identification program reported real cars/tracks, people, boats and other objects. The percentages of each type of target are shown in Figure 22. The large amount of other targets is due to the nature of the search area. The testing site is a large area of cropland near a river, and the local farmers use a type of fertilizer that is stored in blue buckets and they use green nets to fence in their crops. These two item types were reported, as shown in Figure 23. However, these results can be quickly sifted through by the GCS operator. The identification program still reduces the operator's work load, and the search mission was successfully completed in $40 \mathrm{~min}$, beginning when the UAV took off and ending when all of the targets had been reported. 


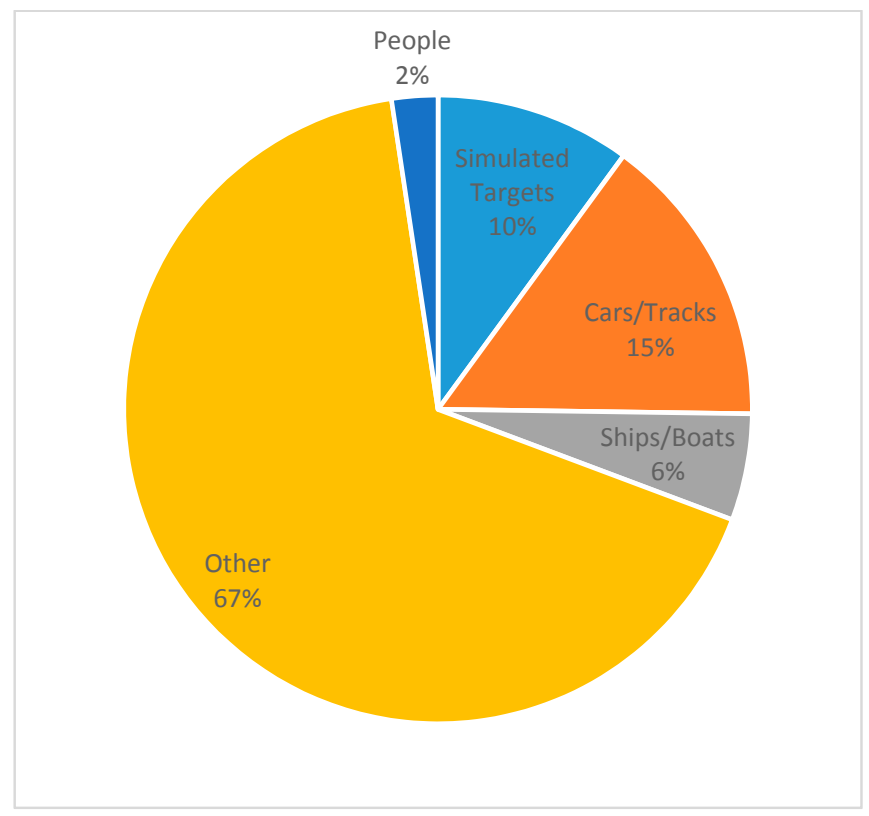

(a)

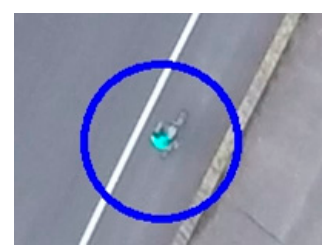

(b)

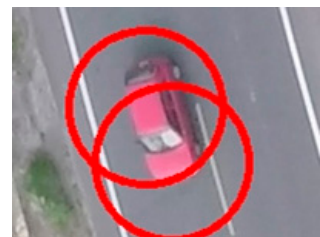

(c)

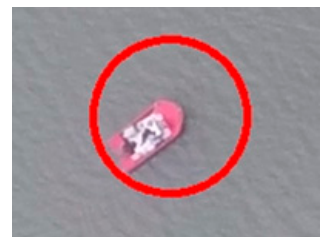

(d)

Figure 22. (a) Composition of reporting targets; (b) a person on the road; (c) a red car and (d) a red boat reported by the identification program.

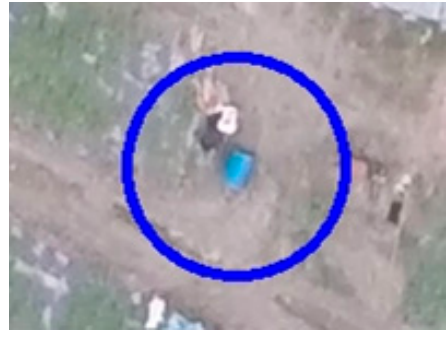

(a)

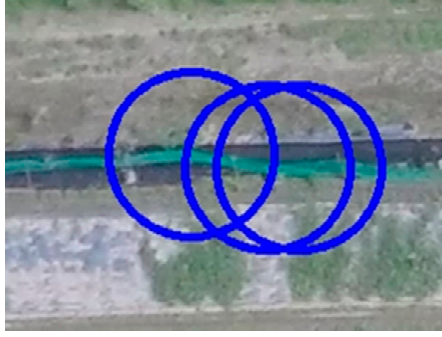

(b)

Figure 23. The other reporting targets: (a) A blue bucket and (b) green nets.

In tests $3-8$, both on-board real-time processing and post-processing were conducted and the results are shown in Figure 24. Note that the performance of the post-target identification is better than that of real-time onboard target identification, due to the higher resolution of the image source. Nevertheless, the on-board target identification system still reported more than $60 \%$ of the targets and provided an efficient real-time supplementary tool for the all-in-one rescue mission. A future study will be conducted to improve the success rates of on-board target identification systems.

\subsection{Mapping}

To cover the whole search area, the flight plan was designed as shown in Figure 25. The distance between 2 adjacent flight paths is $80 \mathrm{~m}$. The total distance of flight plan is $20.5 \mathrm{~km}$ with a flight time of $18 \mathrm{~min}$. The turning radius of the UAV was calculated, and it is $50 \mathrm{~m}$ for bank angles no larger than $35^{\circ}$. Thus, as shown in Figure 25b, the flight plan was designed with a $160-\mathrm{m}$ turning diameter while the gap between the two flight paths remained $80 \mathrm{~m}$ to ensure overlapping and complete coverage.

After the flight, the mapping image capture program developed in this study was applied to capture the images from the high-resolution video and process the flight data log. A total of 2200 photos were generated and applied to Pix4D, and the resulting orthomosaic model and point clouds are shown in Figure 26. The missing part is due to the strong reflection on the water's surface resulting in mismatched features. 


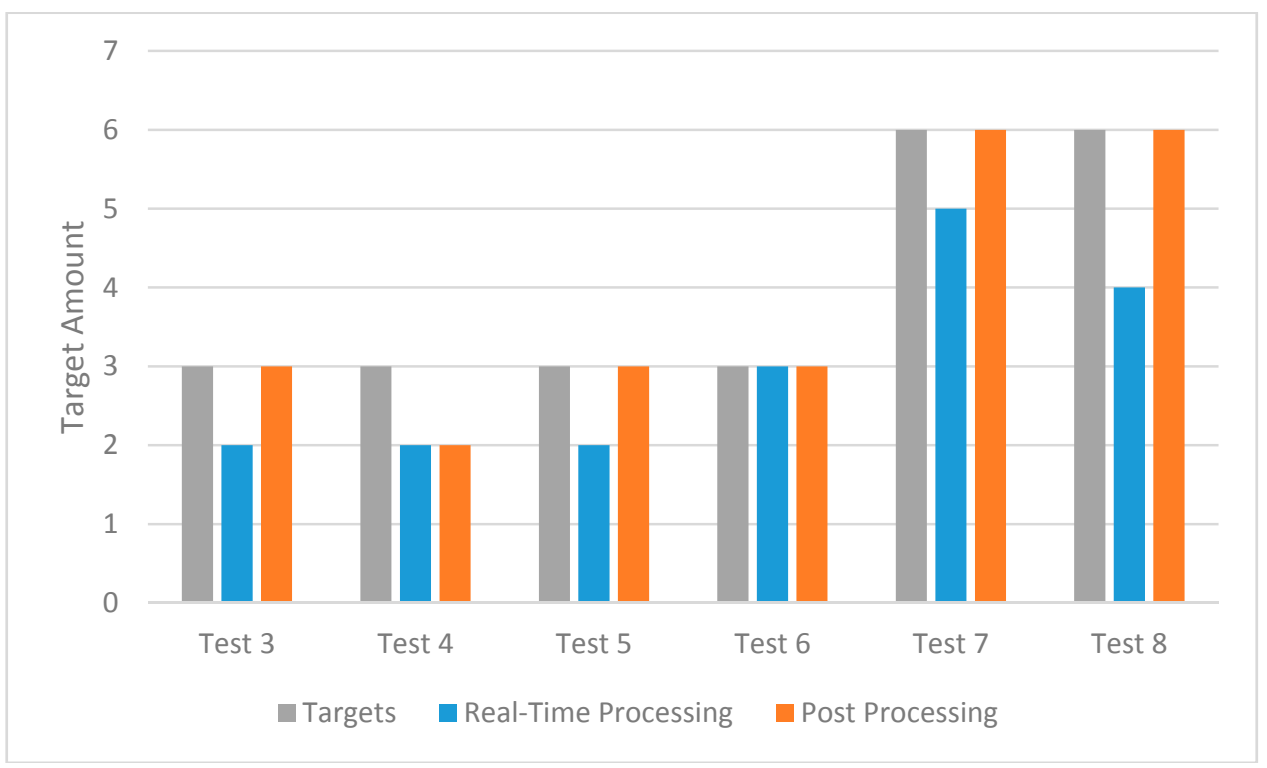

Figure 24. Target identification results of real-time processing and post-processing.

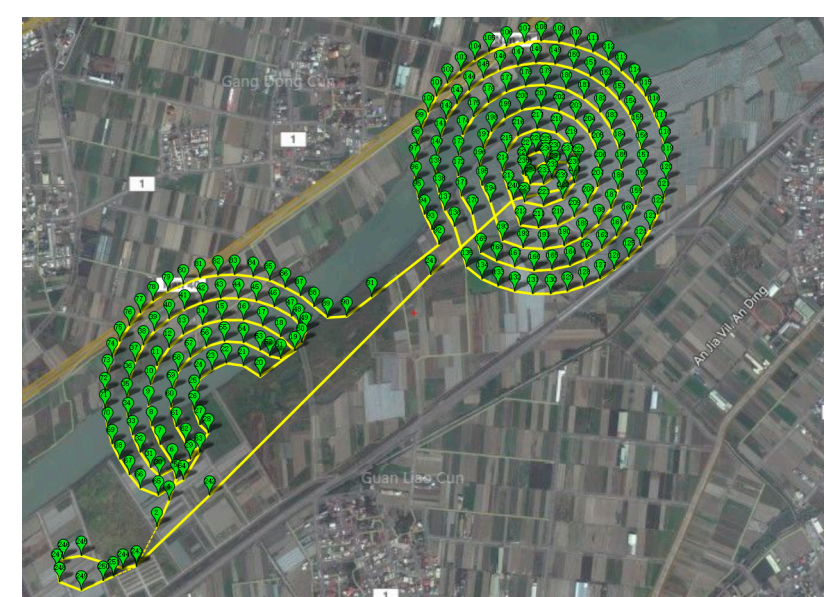

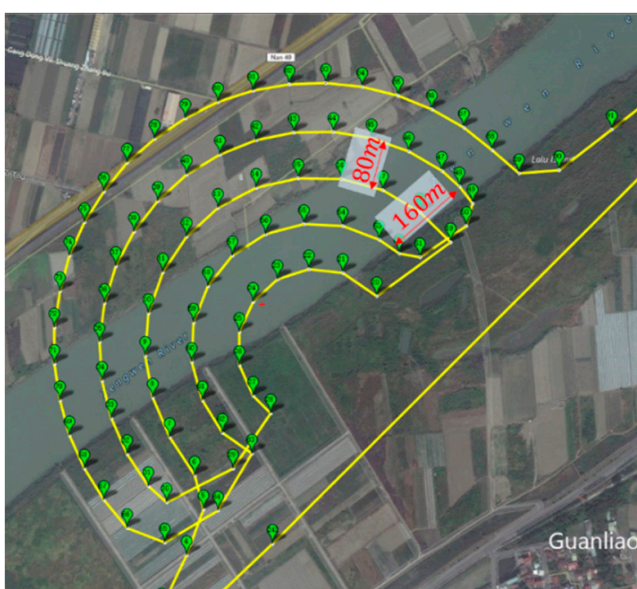

(b)

(a)

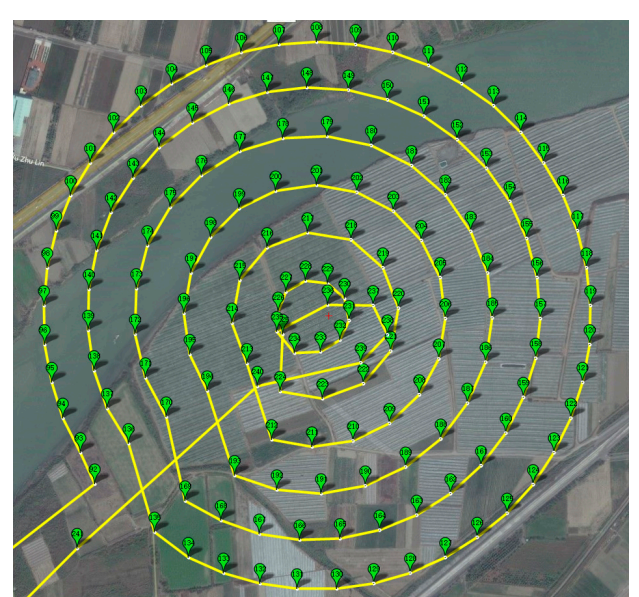

(c)

Figure 25. (a) Overall flight plan for the search mission, (b) flight plan for search area B (the turning diameter reaches $160 \mathrm{~m}$ to ensure the flight performance while the distance between the two flight paths remains $80 \mathrm{~m}$, guaranteeing full coverage and overlap) and (c) flight plan for search area A. 


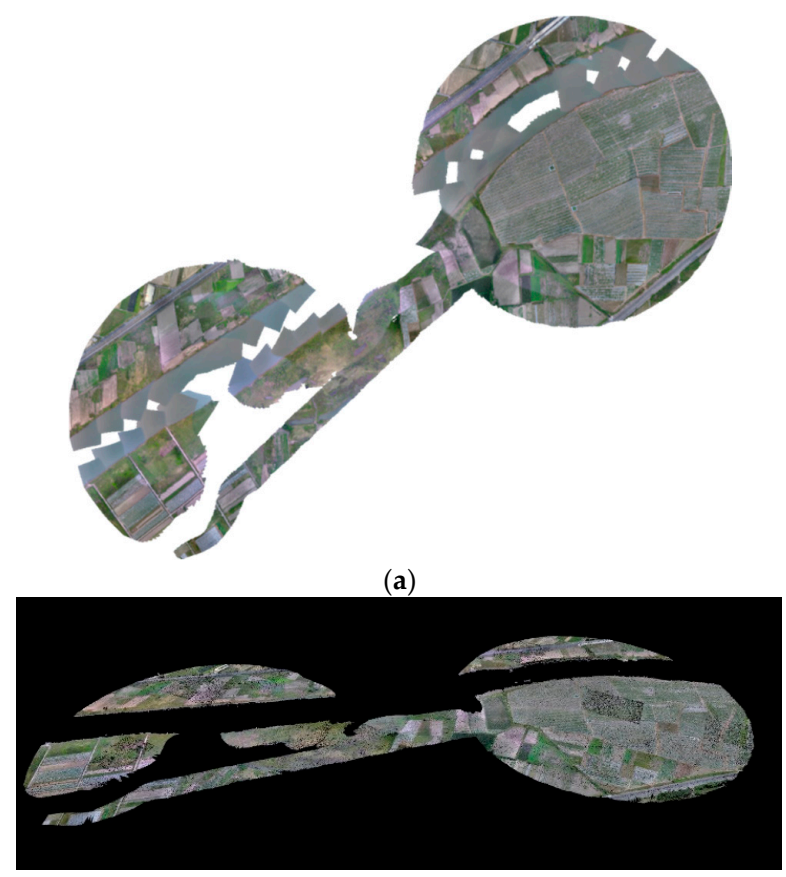

(b)

Figure 26. (a) Orthomosaic model of the testing area and (b) point clouds of the search area.

\section{Conclusions}

In this study, a UAV system was developed, and its ability to assist in SAR missions after disasters was demonstrated. The UAV system is a data acquisition system equipped with various sensors to realize searching and geo-information acquisition in a single flight. The system can reduce the cost of large-scale searches, improve the efficiency and reduce end-users' workloads.

In this paper, we presented a target identification algorithm with a self-adapting threshold that can be applied to a UAV system. Based on this algorithm, a set of programs was developed and tested in a simulated search mission. The test results demonstrated the reliability and efficiency of this new UAV system.

A further study will be conducted to improve the image processing in both onboard and post target identification, focusing on reducing the unexpected reporting targets. A proposed optimization method is to add an extra filtration process to the GCS to further identify the shape of the targets. This proposed method will not increase the computational time of the onboard device significantly. It is a simple but effective method concerning the limited CPU capability of an on-board processor. Generally speaking, most commercial software is too comprehensive to be used in the on-board device. Notably, the limitation of the computing power becomes a minor consideration during post-processing since powerful computing devices can be used at this stage. To evaluate and improve the performance of targets' identification algorithm in post-processing, further study will be conducted, including the application of the parallel computing technology and comparison with the advanced commercial software.

In this study, the scales of the camera and world coordinates were assumed to be linear. This assumption can result in target location errors. We tried to reduce the error by selecting the image with the target near the image center. Although the error of the current system is acceptable for a search mission, we will conduct a further study to improve the location accuracy. Lidar will be installed to replace the sonar, and more accurate relative vehicle height will be provided for auto-landing. Also, in the future, the vehicle will be further integrated to realize the 'Ready-to-Fly' stage for quick responses in real applications. 
Supplementary Materials: The following is available online at https://www.youtube.com/watch? $\mathrm{v}=19$ -RyPp93M. Video S1: A Camera-Based Target Detection and Positioning System for Wilderness Search and Rescue using a UAV. https://github.com/jingego/UAS_system/tree/master/Image\%20Processing. Source Code 1: Matlab Code of targets identification. https://github.com/jingego/UAS_system/blob/master/Mapping_preprocess/CAM_clock_paper_version.m. Source Code 2: MatLab Code of synchronization.

Acknowledgments: This work is sponsored by Innovation and Technology Commission, Hong Kong under Contract No. ITS/334/15FP. Special thanks to Jieming Li for his help in building the image identification algorithm of this work.

Author Contributions: Jingxuan Sun and Boyang Li designed the overall system. In addition, Boyang Li developed the vehicle platform and Jingxuan Sun developed the identification algorithms, locating algorithms and post image processing system. Yifan Jiang developed the on-board targets identification. Jingxuan Sun and Boyang Li designed and performed the experiments. Jingxuan Sun analyzed the experiment results and wrote the paper. Chih-yung Wen is in charge of the whole project management.

Conflicts of Interest: The authors declare no conflict of interest.

\section{Abbreviations}

The following abbreviations are used in this manuscript:

UAV: $\quad$ Unmanned Aerial Vehicle

SAR: $\quad$ Search and Rescue

GCS: $\quad$ Ground Control System

AAT: $\quad$ Auto Antenna Tracker

OSD: $\quad$ On Screen Display

FOV: $\quad$ Field of view

FPS: $\quad$ Frame Per Second

\section{References}

1. Indonesia Airasia Flight 8501. Available online: https://en.wikipedia.org/wiki/Indonesia_Air-Asia_Flight_ 8501 (accessed on 20 October 2016).

2. Qz8501: Body of First Victim Identified. Available online: http://english.astroawani.com/airasia-qz8501news/qz8501-body-first-victim-identified-51357 (accessed on 20 October 2016).

3. Airasia Crash Caused by Faulty Rudder System, Pilot Response, Indonesia Says. Available online: https://www.thestar.com/news/world/2015/12/01/airasia-crash-caused-by-faulty-rudder-systempilot-response-indonesia-says.html (accessed on 20 October 2016).

4. Goodrich, M.A.; Morse, B.S.; Gerhardt, D.; Cooper, J.L.; Quigley, M.; Adams, J.A.; Humphrey, C. Supporting wilderness search and rescue using a camera-equipped mini uav. J. Field Robot. 2008, 25, 89-110. [CrossRef]

5. Goodrich, M.A.; Cooper, J.L.; Adams, J.A.; Humphrey, C.; Zeeman, R.; Buss, B.G. Using a mini-uav to support wilderness search and rescue: Practices for human-robot teaming. In Proceedings of the 2007 IEEE International Workshop on Safety, Security and Rescue Robotics, Rome, Italy, 27-29 September 2007.

6. Goodrich, M.A.; Morse, B.S.; Engh, C.; Cooper, J.L.; Adams, J.A. Towards using unmanned aerial vehicles (UAVs) in wilderness search and rescue: Lessons from field trials. Interact. Stud. 2009, 10, 453-478.

7. Morse, B.S.; Engh, C.H.; Goodrich, M.A. Uav video coverage quality maps and prioritized indexing for wilderness search and rescue. In Proceedings of the 5th ACM/IEEE international conference on Human-robot interaction, Osaka, Japan, 2-5 March 2010.

8. Doherty, P.; Rudol, P. A uav search and rescue scenario with human body detection and geolocalization. In Proceedings of the Australasian Joint Conference on Artificial Intelligence, Gold Coast, Australia, 2-6 December 2007.

9. Habib, M.K.; Baudoin, Y. Robot-assisted risky intervention, search, rescue and environmental surveillance. Int. J. Adv. Robot. Syst. 2010, 7, 1-8.

10. Tomic, T.; Schmid, K.; Lutz, P.; Domel, A.; Kassecker, M.; Mair, E.; Grixa, I.L.; Ruess, F.; Suppa, M.; Burschka, D. Toward a fully autonomous uav: Research platform for indoor and outdoor urban search and rescue. IEEE Robot. Autom. Mag. 2012, 19, 46-56. [CrossRef]

11. Waharte, S.; Trigoni, N. Supporting search and rescue operations with uavs. In Proceedings of the IEEE 2010 International Conference on Emerging Security Technologies (EST), Canterbury, UK, 6-7 September 2010. 
12. Naidoo, Y.; Stopforth, R.; Bright, G. Development of an uav for search \& rescue applications. In Proceedings of the IEEE AFRICON 2011, Livingstone, Zambia, 13-15 September 2011.

13. Bernard, M.; Kondak, K.; Maza, I.; Ollero, A. Autonomous transportation and deployment with aerial robots for search and rescue missions. J. Field Robot. 2011, 28, 914-931. [CrossRef]

14. Cummings, M. Designing Decision Support Systems for Revolutionary Command and Control Domains. Ph. D. Thesis, University of Virginia, Charlottesville, VA, USA, 2004.

15. Olsen, D.R., Jr.; Wood, S.B. Fan-out: Measuring human control of multiple robots. In Proceedings of the SIGCHI Conference on Human Factors in Computing Systems, Vienna, Austria, 24-29 April 2004.

16. Davis, J.W.; Keck, M.A. A two-stage template approach to person detection in thermal imagery. WACV/MOTION 2005, 5, 364-369.

17. Lee, D.J.; Zhan, P.; Thomas, A.; Schoenberger, R.B. Shape-based human detection for threat assessment. In Proceedings of the SPIE 5438, Visual Information Processing XIII, Orlando, FL, USA, 15 July 2004.

18. Mikolajczyk, K.; Schmid, C.; Zisserman, A. Human detection based on a probabilistic assembly of robust part detectors. In European Conference on Computer Vision, Proceedings of the 8th European Conference on Computer Vision, Prague, Czech Republic, 11-14 May 2004; Springer: Berlin/Heidelberg, Germany; pp. 69-82.

19. Rudol, P.; Doherty, P. Human body detection and geolocalization for uav search and rescue missions using color and thermal imagery. In Proceedings of the 2008 IEEE Aerospace Conference, Montana, MT, USA, 1-8 March 2008.

20. Wu, J.; Zhou, G. Real-time uav video processing for quick-response to natural disaster. In Proceedings of the 2006 IEEE International Conference on Geoscience and Remote Sensing Symposium, Denver, CO, USA, 31 July-4 August 2006.

21. Suzuki, T.; Meguro, J.; Amano, Y.; Hashizume, T.; Hirokawa, R.; Tatsumi, K.; Sato, K.; Takiguchi, J.-I. Information collecting system based on aerial images obtained by a small uav for disaster prevention. In Proceedings of the 2007 International Workshop and Conference on Photonics and Nanotechnology, Pattaya, Thailand, 16-18 December 2007.

22. Xi, C.; Guo, S. Image target identification of uav based on sift. Proced. Eng. 2011, 15, 3205-3209.

23. Li, C.; Zhang, G.; Lei, T.; Gong, A. Quick image-processing method of uav without control points data in earthquake disaster area. Trans. Nonferrous Metals Soc. China 2011, 21, s523-s528. [CrossRef]

24. United Eagle Talon Day Fatso FPV Carrier. Available online: http://www.x-uav.cn/en/content/?463.html (accessed on 20 October 2016).

25. Hardkernel Co., Ltd. Ocam: 5mp USB 3.0 Camera. Available online: http:/ /www.hardkernel.com/main/ pro-ducts /prdt_info.php?g_code=G145231889365 (accessed on 20 October 2016).

26. Chen, Y.; Hsiao, F.; Shen, J.; Hung, F.; Lin, S. Application of matlab to the vision-based navigation of UAVs. In Proceedings of the 2010 8th IEEE International Conference on Control and Automation (ICCA), Xiamen, China, 9-11 June 2010.

27. Gopro hero4 Silver. Available online: http://shop.gopro.com/APAC/cameras/hero4-silver/CHDHY-401EU.html (accessed on 20 October 2016).

28. Hero3+ Black Edition Field of View (FOV) Information. Available online: https://gopro.com/support/ articles-/hero3-field-of-view-fov-information (accessed on 20 October 2016).

(C) 2016 by the authors; licensee MDPI, Basel, Switzerland. This article is an open access article distributed under the terms and conditions of the Creative Commons Attribution (CC-BY) license (http://creativecommons.org/licenses/by/4.0/). 